\title{
A Survey of U.S. Commercial Environmental and Industrial Optical Sensor Environmental Monitoring Equipment Manufacturers
}

\author{
Topical Report \\ October 29, 1998

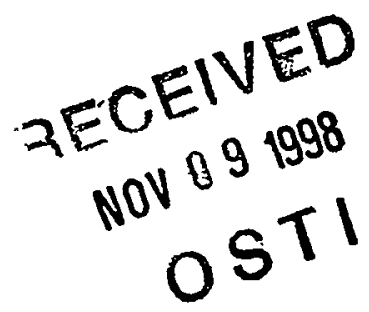

Work Performed Under Contract No.: DE-FC21-94MC31179

\author{
For \\ U.S. Départment of Energy \\ Office of Fossil Energy \\ Federal Energy Technology Center \\ P.O. Box 880 \\ Morgantown, West Virginia 26507-0880
}

By

Global Environment \& Technology Foundation

7010 Little River Turnpike

Suite 300

Annadale, Virginia 22003 


\section{Disclaimer}

This report was prepared as an account of work sponsored by an agency of the United States Government. Neither the United States Government nor any agency thereof, nor any of their employees, makes any warranty, express or implied, or assumes any legal liability or responsibility for the accuracy, completeness, or usefulness of any information, apparatus, product, or process disclosed, or represents that its use would not infringe privately owed rights. Reference herein to any specific commercial product, process, or service by trade name, trademark, manufacturer, or otherwise does not necessarily constitute or imply its endorsement, recommendation, or favoring by the United States Government or any agency thereof. The views and opinions of authors expressed herein do not necessarily state or reflect those of the United States Government or any agency thereof. 


\section{DISCLAIMER}

Portions of this document may be illegible in electronic image products. Images are produced from the best available original document. 
Fourier Transform InfraRed (FTIR)

Spectrometers - Open Path and Extractive 


\section{Analect Instruments, Inc.}

\section{Address:}

9420 Jeronimo Rd.

Irvine, $\mathrm{CA}, \underline{92618}$

Tel: (714) 206-4100; (800) 326-2328

Fax: (714) 206-4120

\section{Internet:}

Explore: Resource Pages

HomePage: http://www.analect.com

Status:

Independent private U.S. company. Formed in 1996.

Size:

- Empl-30 employees; the employee growth rate for the year prior to interview is unknown; $33 \%$ employment growth projected for the next fiscal year.

- Sales - $\$ 5 \mathrm{~m}-\$ 10$ annual sales; $10 \%$ to $25 \%$ of sales are from international business.

Execs:

- John Morrison, Chief Executive Officer (C.E.O.)

- James Malone, VP of Sales (Sales)

- George Moore, VP of Operations (Engineering)

- Burce McIntosh, VP of Technology (Corporate Dev., Tech Transfer)

- Jim Mowry, Chief Financial Officer (Finance)

- Bill Brodley, Marketing Director (Marketing)

- Rocco Guerriero, Manufacturing Manager (Manufacturing)

- Ms. Melanie Kabana, Office Manager (Administration)

Primary Industry: Test \& Measurement (TAM)

Primary SIC Code: 3820

Mânufacturer of Fourier transform infrared (FTIR) process monitoring systems. Products are sold to multiple industries.

Product Codes:

- $\underline{\mathrm{TAM}}-\underline{\mathrm{SC}}-\underline{\mathrm{ISF}} . . .$. . Fourier transform infrared process monitoring systems - (SIC Code - $\underline{3826}$ ) 


\section{ASI Applied Systems, LLC}

\section{Address:}

8223 Cloverleaf Dr., Suite 102

Millersville, MD 21108

Tel: (410) $987-3222$

Fax: (410) 987-2626

\section{Internet:}

Explore: Resource Pages

e-mail: henrydubina@asirxn.com

\section{Status:}

Independent private U.S. company. Formed in 1990.

Size:

- Empl - 35 employees; $34 \%$ employment growth in the year prior to interview; $2 \%$ employment growth projected for the next fiscal year.

- Sales - $\$ 5 \mathrm{~m}$ - $\$ 10$ annual sales; over $25 \%$ of sales are from international business.

Execs:

- Henry Dubina, President (C.E.O., Sales)

- Dr. Alan Rein, VP of Technology (R\&D, Marketing)

- Ms. Nancy Hutnik, Director of Finance (Finance, Administration)

- Dr. Jeff Sherman, Director of Intemational Sales (Int'l Sales \& Mktg.)

- Morris Martelle, Manufacturing Manager (Manufacturing)

- Peter Bennett, Engineering Manager (Engineering)

Primary Industry: Test \& Measurement (TAM)

Primary SIC Code: 3820

Designer and manufacturer of Fourier transform infrared spectroscopy (FTIR) based monitoring equipment.

Products are used for monitoring reactions in biological and chemical processes. Products are sold to the chemical, .pharmaceutical, polymer and biotechnology industries. This company was capitalized by private investment.

Product Codes:

- $\underline{\text { IAM }}$ - $\underline{\text { SC }}$ - ISF ...... Fourier transform infrared spectrometers - (SIC Code - 3826) 


\section{Axiom Analytical, Inc.}

\section{Address:}

18103-C Sky Park South

Irvine, $\mathrm{CA} 92714$

Tel: (714) 757-9300

Fax: (714) 757-9306

\section{Internet:}

Explore: Resource Pages

HomePage: http://www.goaxiom.com/axiom

e-mail: goaxiom@aol.com

\section{Status:}

Independent private U.S. company. Formed in 1988.

Size:

- Empl- 6 employees; $20 \%$ employment growth in the year prior to interview; $33 \%$ employment growth projected for the next fiscal year.

- Sales - $\$ 1 \mathrm{~m}-\$ 2.5 \mathrm{~m}$ annual sales; $10 \%$ to $25 \%$ of sales are from international business.

\section{Execs:}

- Walter M. Doyle, President (C.E.O., R\&D)

- Norman Jennings, Executive Vice President (Sales, Marketing, Finance)

\section{Primary Industry: Test \& Measurement (TAM)}

\section{Primary SIC Code: 3820}

Manufacturer of fourier transform near infrared spectrometry equipment, immersion probes, flow liquid monitors, wastewater analysis equipment and stack and corrosive gas analysis equipment. Products are sold to FTIR spectrometer manufacturers and the general petrochemical industry.

\section{Product Codes:}

$-0 \underline{A U T}-\underline{A T}-\underline{A}$...... Immersion probes - (SIC Code - 3829).

- ENV - AN - G ...... Gas analysis equipment - (SIC Code - 3823)

- ENV - $\underline{\mathrm{AN}}-\underline{\mathrm{W}} . . . .$. . Wastewater analysis equipment - (SIC Code $-\underline{3823}$ )

- TAM - ME - I ..... . Flow liquid monitors - Tunnel CellTM - (SIC Code - 3825)

- TAM - ME - MF ...... Flow measuring equipment - (SIC Code - 3823)

- $\underline{\text { TAM }}$ - $\underline{\text { SC }}$ - ISF ...... FTIR spectrometer equipment - (SIC Code - $\underline{\text { 3826) }}$ 


\section{-Bio-Rad Laboratories, Inc. / Digilab Division}

- FKA: Bio-Rad Laboratories, Inc. / Spectroscopy Division

Address:

237 Putnam Ave.

Cambridge, MA 02139

Tel: (617) 868-4330; (800) 225-1248

Fax: (617) 234-7043

\section{Internet:}

Explore: Resource Pages

HomePage: http://www.digilab.bio-rad.com

e-mail: sales.digilab@bio-rad.com

\section{Status:}

Operating unit of a public U.S. company (Ticker symbol: BIOA). This operating unit formed in 1970. Ultimate parent is Bio-Rad Laboratories, Inc.

Government Contractor: Prime

Size:

- Empl - 155 employees; no employment change in the year prior to interview; no employment change projected for the next fiscal year.

- Sales - $\$ 100 \mathrm{~m}-\$ 250 \mathrm{~m}$ annual sales; over $25 \%$ of sales are from international business.

\section{Execs:}

- David Schwartz, President/CEO (C.E.O.)

- Bob Kynoch, Financial Controller (Finance)

- Raul Curbelo, Director of Engineering (Engineering, R\&D)

- Ms. Karen Mahoney, Director of Purchasing (Purchasing)

- Jerry Keahl, Division Manager

- Ron Sostek, Marketing Manager (Marketing)

- ० Dr. K. Krishnan, International Sales Manager (Int'l Sales \& Mktg.)

- Joe Griffin, Manufacturing Manager (Manufacturing)

- Richard Crocombe, Product Manager

- Ms. K. Aluia, Personnel Administrator (Personnel)

- John McDonald, National Sales Manger (Sales)

\section{Primary Industry: Test \& Measurement (TAM)}

Primary SIC Code: 3820

Manufacturer of Fourier transform infrared spectrometers and accessories. Products are sold to the chemical, pharmaceutical, biotech, energy and materials industries. This company was capitalized by private investment.

\section{Product Codes:}

- $\underline{\mathrm{TAM}}$ - $\underline{\mathrm{SC}}-\underline{\mathrm{ISF}}$...... Fourier transform infrared spectrometers - (SIC Code - $\underline{3826}$ ) 


\section{Bruker Instruments, Inc.}

- PMW: IBM Instruments, Inc.

\section{Address:}

19 Fortune Dr., Manning Park

Billerica, MA $\underline{01821}$

Tel: (508) 667-9580

Fax: (508) 667-3954

\section{Internet:}

Explore: $\underline{\text { Resource Pages }}$

HomePage: http://www.bruker.com

\section{Status:}

Operating unit of a non-U.S. parent. This operating unit formed in 1972. Ultimate parent is Bruker Analytik Nesstechnik (country Germany).

Size:

- Empl - 150 employees; no employment change in the year prior to interview; no employment change projected for the next fiscal year.

- Sales - Estimated annual sales $=\$ 20$ million; under $2.5 \%$ of sales are from international business.

Execs:

- Guenther Laukien, President (Chairman, C.E.O.)

- Dr. Frank Laukien, Executive Vice President (Finance, Sales, Marketing)

- Dr. Dirk Laulien, Vice President

\section{Primary Industry: Test \& Measurement (TAM)}

Primary SIC Code: 3820

Manufacturer of laboratory instruments specializing in magnetic resonance imaging equipment, programmable power supplies, infrared spectrometers, mass mobile spectrometers, nuclear mobile spectrometers and electro paramagnetic resonance equipment. Products are sold to scientific laboratories.

\section{Product Codes:}

- MED - DG - IR ...... Magnetic resonance imaging equipment - (SIC Code - 3845)

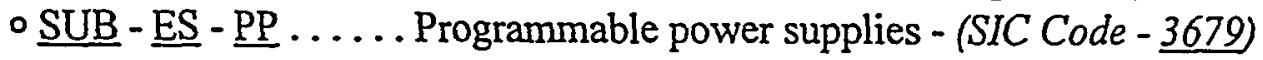

- $\underline{\text { TAM }}$ - $\underline{\text { SC }}$ - ISF ...... Infrared spectrometers - (SIC Code - 3826)

- $\underline{\text { TAM }}$ - $\underline{\mathrm{SC}}-\underline{\mathrm{ISM}}$....... Mass mobile spectrometers - (SIC Code - $\underline{3826)}$

- TAM - $\underline{\text { SC }}-\underline{\text { ISN }}$...... Electro paramagnetic resonance equipment - (SIC Code $-\underline{3829}$ )

- $\underline{\mathrm{TAM}}$ - $\underline{\mathrm{SC}}-\underline{\text { ISN }} . . . .$. . Nuclear magnetic resonance spectrometers - (SIC Code $-\underline{3829}$ ) 


\section{Environmental Technologies Group, Inc.}

- FKA: AlliedSignal Inc. / Bendix Environmental Systems Division

\section{Address:}

1400 Taylor Ave., PO Box 9840

Baltimore, MD 21286

Tel: (410) 321-5200; (800) 635-4598

Fax: (410) $321-5255$

\section{Internet:}

Explore: $\underline{\text { Resource Pages }}$

\section{- Status:}

Independent private U.S. company. Formed in 1988.

Government Contractor: Prime \& Sub-contract

Size:

- Empl - 150 employees; no employment change in the year prior to interview; no employment change projected for the next fiscal year.

- Sales - $\$ 25 \mathrm{~m}-\$ 50 \mathrm{~m}$ annual sales; $10 \%$ to $25 \%$ of sales are from international business.

\section{Execs:}

- Dr. Timothy Karpetsky, President (C.E.O.)

$\circ$ R. Morin, VP of Finance (Finance)

- Sam Ankerbrandt, VP of Marketing (Sales, Marketing)

- Rick Thomas, VP of Engineering (Engineering, R\&D)

- Rick Priddy, VP of Operations (Manufacturing)

- William Morrison, Manager of M.I.S. (M.I.S.)

Primary Industry: Defense (DEF)

Primary SIC Code: 8700

Manufacturer of meteorological systems for the military. The systems are used to provide first round artillery effectiveness predictions, chemical and nuclear fall-out predictions and weather forecasting. The chemical sensors - are used to detect hazardous chemicals, such as those found on the battlefield. This company was capitalized by private investment.

\section{Product Codes:}

- $\underline{D E F}-\underline{G R}-\underline{Z} \ldots .$. Meteorological military systems - (SIC Code $-\underline{3799})$

- $\underline{\mathrm{DEF}}-\underline{\mathrm{SV}}$...... Meteorological systems integration services - (SIC Code $-\underline{8700})$

- $\underline{\mathrm{DEF}}-$ WA $\ldots . .$. Artillery effectiveness predictions - (SIC Code $-\underline{3812}$ )

- ENR - ME ..... . Ground-based meteorological systems - (SIC Code - 3829)

- $\underline{E N V}-\underline{A N}-\underline{G}$...... Chemical sensors and alarms - (SIC Code - 3823)

- TAM - DE ...... Chemical detection systems - (SIC Code - 3820) 


\section{Idealab, Inc.}

\section{Address:}

305 Union St., PO Box 427

Franklin. MA 02038

Tel: (508) 528-9260

Internet:

-Explore: $\underline{\text { Resource Pages }}$

Status:

Independent private U.S. company. Formed in 1960.

Size:

- Empl - 14 employees; no employment change in the year prior to interview.

- Sales - Estimated annual sales $=\$ 1.8$ million; no international sales.

Execs:

- James Pritchard, President (C.E.O., Sales, Finance, R\&D, Purchasing, Personnel)

- Ed Gunter, Managing Director (Manufacturing)

- Martin Lantery, Marketing Manager (Marketing)

Primary Industry: Test \& Measurement (TAM)

Primary SIC Code: 3820

Manufacturer of fourier transform infrared (FTIR) spectrometer systems. Products are sold to the government, universities and test and measurement laboratories.

\section{Product Codes:}

- $\underline{\text { TAM }}$ - $\underline{\mathrm{SC}}-\underline{\mathrm{ISF}}$...... Fourier transform infrared (FTIR) spectrometer systems - (SIC Code $-\underline{3826}$ ) 


\section{Laser Photonics, Inc. / Analytics Division}

- FKA: Spectra-Physics, Inc. / Laser Analytics Division

Address:

10 Upton Dr.

Wilmington, MA 01887

Tel: (508) 658-9100; (800) 344-0621

Fax: (508) 658-1888

\section{Internet:}

Explore: Resource Pages

e-mail: diodelaser@aol.com

Status:

Operating unit of a public U.S. company (Ticker symbol: ZAPP). This operating unit formed in 1975. Ultimate parent is Helionetivs, Inc.

Government Contractor: Prime \& Sub-contract

Size:

- Empl - 9 employees; $31 \%$ employment reduction in the year prior to interview; $22 \%$ employment growth projected for the next fiscal year.

- Sales - Annual sales $=\$ .80$ million; over $25 \%$ of sales are from international business.

\section{Execs:}

- Edward Renfer, General Manager (C.E.O., Finance)

- Ze'ev Feit, Laser Research and Development Director (R\&D)

- John Swierz, Sales and Marketing Manager (Sales, Marketing)

- Jon Sproul, Engineering Manager (Engineering)

Primary Industry: Photonics (PHO)

Primary SIC Code: 3699

Designer and manufacturer of tunable infrared lead-salt diode lasers, high resolution laser source spectrometers, photodetectors and multipass cells. The products are used $80 \%$ for scientific and research work with the remaining $20 \%$ used in industrial applications. Products are sold to multiple industries.

\section{Product Codes:}

- $\underline{\mathrm{PHO}}-\underline{\mathrm{LA}}-\underline{\mathrm{LMZ}}$...... Tunable infrared lead-sait diode lasers - (SIC Code $-\underline{3699}$ )

- $\underline{\text { PHO }}-\underline{\mathrm{OE}}-\underline{\mathrm{D}}$....... Photodetectors - (SIC Code - $\underline{3679)}$

- $\underline{\mathrm{PHO}}-\underline{\mathrm{OE}}-\underline{\mathrm{EZ}}$....... Multipass cells - (SIC Code - $\underline{3674}$ )

- $\underline{\text { TAM }}-\underline{\text { SC }}-\underline{\text { ISF }} . . .$. . . High resolution laser source spectrometers - (SIC Code $-\underline{3826}$ ) 


\section{Mattson Unicam Cahn}

- FKA: ATI Instruments North America

- PMW: Cahn Instruments, Inc.

\section{Address:}

1001 Fourier Dr.

Madison, WI 53717

Tel: (608) 831-5515

Fax: (608) 831-2093

\section{Internet:}

Explore: Resource Pages

\section{Status:}

Operating unit of a public U.S. company (Ticker symbol: TMO). This operating unit formed in 1983. Ultimate parent is Thermo Electron Corp.

Government Contractor: Prime

Size:

- Empl - 65 employees; $37 \%$ employment reduction in the year prior to interview; no employment change projected for the next fiscal year.

- Sales - Annual sales $=\$ 15$ million; over $25 \%$ of sales are from international business.

Execs:

- Zbiengnie Stanek, General Manager (C.E.O., Marketing)

- Ms. Dawn Leeder, Controller (Finance)

- Paul Hansen, Director of Operations (Manufacturing, R\&D)

- Don Mills, Director of M.I.S. (M.I.S.)

- Paul Hinahara, Engineering Manager (Engineering)

Primary Industry: Test \& Measurement (TAM)

Primary SIC Code: 3820

Manufacturer of spectrometers and lab scales and balances. The spectrometers are sold to industrial firms, academic institutions and to the U.S. government for the purpose of identifying microscopic materials. The company also - develops FTIR spectrometry software for MS-DOS and Macintosh systems.

\section{Product Codes:}

- $\underline{\text { SOF }}-\underline{\mathrm{HL}}-\underline{\mathrm{L}} \ldots . .$. FTIR spectrometry software - WinFIRSTTM $-($ SIC Code $-\underline{7372}$ )



- $\underline{\text { TAM }}-\underline{\text { SC }}$ - ISF ...... Fourier transform infrared spectrometers - (SIC Code - 3826)

- $\underline{\mathrm{TAM}}-\underline{\mathrm{SC}}-\underline{\mathrm{SS}}$....... Lab scales and balances - (SIC Code - $\underline{3821})$ 


\section{MIIDAC Corp.}

\section{Address:}

17911 Fitch Ave.

Irvine, CA 92714

Tel: (714) 660-8558

Fax: (714) 660-9334

\section{Internet:}

Explore: Resource Pages

HomePage: http://www.midac.com

e-mail:info@midac.com

\section{Status:}

Independent private U.S. company. Formed in 1978.

\section{Government Contractor: Prime}

- Size:

- Empl- 30 employees; no employment change in the year prior to interview.

- Sales - $\$ 5 \mathrm{~m}$ - $\$ 10$ annual sales; over $25 \%$ of sales are from international business.

\section{Execs:}

- Gerald Auth, President (C.E.O., R\&D)

- Ms. Carol Auth, Operations Manager (Finance, Administration, Personnel)

- Scott Fleming, General Manager (Manufacturing)

- Ms. Heidi Auth, Sales and Marketing Manager (Sales, Marketing)

- Ray Milward, International Sales Manager (Int'l Sales \& Mktg.)

- Ms. Margo Amann, Purchasing Agent (Purchasing)

\section{Primary Industry: Test \& Measurement (TAM)}

Primary SIC Code: 3820

Manufacturer of Fourier transform infrared spectrometer systems. The systems are used to analyze solids, liquids and gases and are primarily used by universities, quality control and forensic laboratories, the pharmaceutical industry and government for research.

Product Codes:

- $\underline{\text { TAM }}$ - $\underline{\text { SC }}$ - $\underline{\text { ISF }} . . . .$. Fourier transform infrared spectrometer systems - (SIC Code - $\underline{\text { 3826) }}$ 


\section{Nicolet Instrument Corp.}

- PMW: Nicolet Instrument Corp. / Test Instruments Division

- PMW: Nicolet Instrument Corp. / Analytical Division

\section{Address:}

5225 Verona Rd.

Madison. WI 53711

Tel: (608) 276-6100; (800) 232-1472

Fax: (608) 273-5046

Internet:

Explore: Resource Pages

HomePage: http://www.nicolet.com

e-mail: nicinfo@nicolet.com

Status:

Operating unit of a public U.S. company (Ticker symbol: TMO). This operating unit formed in 1967. Ultimate parent is Thermo Electron Corp.

Government Contractor: Prime \& Sub-contract

Size:

- Empl - 450 employees; no employment change in the year prior to interview; no employment change projected for the next fiscal year.

- Sales - Estimated annual sales $=\$ 60$ million; over $25 \%$ of sales are from international business.

\section{Execs:}

- Robert J. Rosenthal, President (C.E.O.)

- John N. Hatsopoulos, Chief Financial Officer (Finance)

- Stephen R. Lowry, Vice President

- Dale C. Davig, Vice President

- Ms. Brenda Wilcox, Controller

- o Jonathan W. Painter, Treasurer

- Denis A. Helm, Director

- Earl R. Lewis, Director

- Arvin H. Smith, Director

- Ms. Sandra L. Lambert, Secretary

- Robert V. Aghababian, Assistant Secretary

- Seth H. Hoogasian, Assistant Secretary

- Paul F. Kelleher, Assistant Secretary

Primary Industry: Test \& Measurement (TAM)

Primary SIC Code: 3820

Manufacturer of fourier transform infrared (FT-IR) and FT-raman spectrometers; microscopes, waveform analyzers and acquisition equipment; and chemical analysis equipment. Products are used by the chemical, pharmaceutical, polymer, petrochemical, food and energy industries and by the government and university laborotories to perform a variety of chemical analyses. 


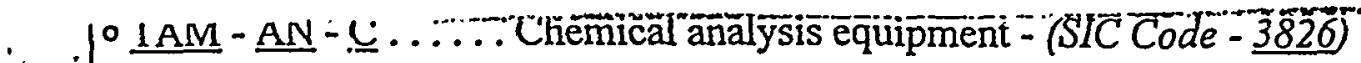

. TAM - AN - OW ...... Waveform analyzers - (SIC Code - 3825)

- $\underline{\mathrm{TAM}}-\underline{\mathrm{CR}}-\underline{\mathrm{D}} \ldots . .$. Waveform acquisition equipment - (SIC Code - 3823)

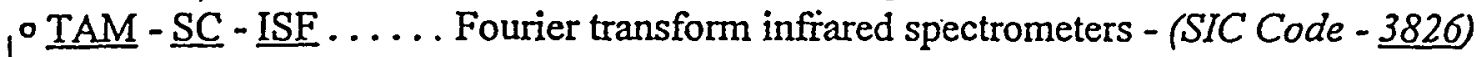

. $\underline{\text { IAM }}$ - SC - ISF ..... Fourier transform-raman spectrometers - (SIC Code - 3826) 


\section{Radian International, LLC}

- FKA: Radian Corp.

- PMW: Dow Environmental, Inc.

\section{Address:}

PO Box 201088

Austin, TX 78720

Tel: (512) 454-4797

Fax: (512) 419-6282

\section{Internet:}

Explore: Resource Pages

HomePage: http://www.radian.com/

\section{Status:}

Operating unit of a public U.S. company (Ticker symbol: HSB). This operating unit formed in 1975. Ultimate parent is The Hartford Steam Boiler Inspection and Insurance Co.

Government Contractor: Prime \& Sub-contract

Size:

- Empl-2,400 employees; no employment change in the year prior to interview; no employment change projected for the next fiscal year.

- Sales - $\$ 100 \mathrm{~m}-\$ 250 \mathrm{~m}$ annual sales; over $25 \%$ of sales are from international business.

\section{Execs:}

- Dr. Donald Carlton, President (C.E.O.)

- Murray Wells, VP of International Marketing (Int'l Sales \& Mktg., Sales, Marketing)

- Dr. P.E. Hudson, Executive VP of Corporate Operations (Finance, Purchasing, Corporate Dev.)

- W. Neal Kocurek, Executive VP of Technical Staff (R\&D, M.I.S., Personnel)

- Scott LaGrone, Senior Vice President (Administration)

- Lowell R. Edmonson, VP of Contracts

- Paul Goldstein, Senior VP of Remediation

- Ms. Cheryl Goad, Communications Manager

\section{Primary Industry: Environmental (ENV)}

Primary SIC Code: 8700

Provider of waste management and consulting services, site, groundwater and soil remediation services, research and development services in glass and ceramics, material and metallurgy testing services, chemical consulting services, computer systems analysis services and manufacturing process consulting services. The company also develops expert systems development, chemical engineering reporting, hazardous chemical gas tracking and oil : company mapping software. The software is IBM PC compatible. This company is a joint venture between The Dow Chemical Company and Hartford Steam Boiler Inspection \& Insurance Company. Products and services are sold to multiple industries.

Product Codes:

- $\underline{\mathrm{CHE}}-\underline{\mathrm{SV}}-\underline{\mathrm{C}} \ldots . .$. Chemical consulting services - (SIC Code $-\underline{8742)}$.

- $\underline{\mathrm{COM}}-\underline{\mathrm{SV}}-\underline{\mathrm{CA}}$...... Computer systems analysis services - (SIC Code - 1 8742)

- $\underline{\mathrm{ENV}}-\underline{\mathrm{SV}}-\underline{\mathrm{CW}} \ldots . .$. Waste management consulting services - (SIC Code - 8742)

- $\underline{E N V}-\underline{\mathrm{SV}}-\underline{\mathrm{WT}} \ldots .$. . Soil remediation services - (SIC Code - 4959) 
- ENV - $\underline{\text { SV }}-\underline{\text { WT }}$...... Groundwater remediation services - (SIC Code - 4959)

- ENV - SV - WT ...... Site remediation services - (SIC Code - 4959)

- ENV - SV - WT ...... Waste management services - (SIC Code - 4959)

- $\underline{\text { MAN }}-\underline{\mathrm{SV}}-\underline{\mathrm{C}}$...... Manufacturing process consulting services - (SIC Code $-\underline{8742}$ )

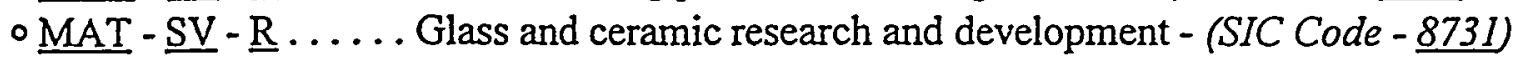

- MAT - SV - T ....... Material and metallurgy testing services - (SIC Code - $\underline{8734)}$

- $\underline{\mathrm{SOF}}-\underline{\mathrm{AI}}-\underline{\mathrm{EC}}$...... Expert systems development software - (SIC Code - 7372)

- $\underline{\text { SOF }}-$ TS - EA ...... Chemical engineering reporting software - (SIC Code - 7372)

- $\underline{\mathrm{SOF}}-\mathrm{TS}-\underline{\mathrm{EA}}$...... Hazardous chemical gas tracking software - (SIC Code - $\underline{7372)}$

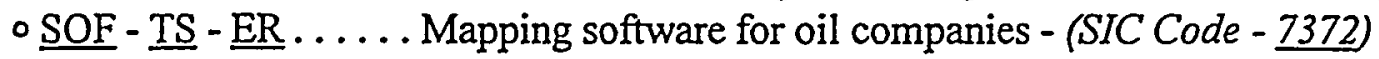




\section{Spectra Optics, Inc. / Block Engineering Division}

- AKA: Block Engineering

\section{Address:}

164 Locke Dr.

Marlborough, MA 01752

Tel: (508) 480-9643

Fax: (508) 480-9226

Internet:

Explore: Resource Pages

HomePage: http://www.members.aol.com/blockeng

\section{Status:}

Operating unit of an independent private U.S. company. This operating unit formed in 1960. Ultimate parent is Spectra Optics. Inc.

Government Contractor: Prime

Size:

- Empl - 10 - 24 employees; the employee growth rate for the year prior to interview is unknown.

- Sales $-\$ 1 \mathrm{~m}-\$ 2.5 \mathrm{~m}$ annual sales; $10 \%$ to $25 \%$ of sales are from international business.

\section{Execs:}

- E. Robert Schildkraut, General Manager (C.E.O.)

- Ms. Virginia Morlock, Director of Marketing (Marketing)

- Tom Quinn, Director of Programs (Engineering)

Primary Industry: Test \& Measurement (TAM)

Primary SIC Code: 3820

Manufacturer of custom and off-the-shelf fourier transform infrared spectrometers and radiometers. The products are used to detect remote gases for identification purposes in defense and surveillance applications. Products are sold to the government. This company was capitalized by private investment.

\section{Product Codes:}

- $\underline{\mathrm{PHO}}-\underline{\mathrm{OP}}-\underline{\mathrm{IR}}$...... Radiometers - (SIC Code - $\underline{3826}$ )

- IAM - $\underline{\text { SC }}$ - ISF...... Fourier transform infrared spectrometers - (SIC Code - $\underline{3826)}$

- $\underline{\text { TAM }}$ - $\underline{\mathrm{SS}}-\underline{\mathrm{SG}} . . . .$. . Gas detection equipment - (SIC Code - $\underline{3669})$ 
RAMAN LASER SYSTEMS 
Aerodyne Research, Inc.

\section{Address:}

45 Manning Rd.

Billerica, MA 01821

Tel: (508) 663-9500

Fax: (508) 663-4918

\section{Internet:}

Explore: Resource Pages

e-mail: corp@aerodyne.com

\section{Status:}

Independent private U.S. company. Formed in 1970.

Government Contractor: Prime \& Sub-contract

Size:

- Empl - 46 employees; $5 \%$ employment reduction in the year prior to interview; $4 \%$ employment growth projected for the next fiscal year.

- Sales - Annual sales $=\$ 6$ million; $2.5 \%$ to $10 \%$ of sales are from international business .

\section{Execs:}

- John H. Kellogg, Chairman of the Board (Chairman)

- Dr. Charles E. Kolb, President/CEO (C.E.O., R\&D)

- Dr. David B. Stickler, Executive Vice President (Engineering)

- George N. Wittreich, Vice President/CFO (Finance)

- Dr. Herman E. Scott, Executive Vice President (Sales)

- James W. Akimchuk, Purchasing Director (Purchasing)

- Ms. Laurie Dean, Communications Manager (Manufacturing)

- Ms. Emily Pruyn, Personnel Administrator (Personnel, Administration)

Primary Industry: Defense (DEF)

Primary SIC Code: 8700

Provider of contract research, development and systems analysis services in remote sensing, surveillance, monitoring and tracking for military aerospace, environmental, industrial process and combustion systems. Provider of laboratory and theoretical research services in optics, electro-optics, chemical physics, combustion, surface and materials science. Manufacturer of point source and remote monitoring systems for atmospheric and environmental trace species utilizing advanced laser and spectroscopic techniques, spectral monitors for combustion systems, laser probes and spectral monitors for chemical and plasma deposition, plasma etching reactors for electronic materials fabrication, optical computing and optical signal processing circuits and algorithms and advanced computer models of spectral signatures for military and aerospace systems. Products and services are sold to multiple industries. This company was capitalized by private investment.

\section{Product Codes:}

- $\underline{\mathrm{CHE}}$ - $\underline{\mathrm{SV}}-\underline{\mathrm{R}}$...... Laboratory and theoretical research services in chemical physics - (SIC Code - 8731 )

$\circ \underline{\mathrm{DEF}}-\underline{\mathrm{SV}} \ldots . . \mathrm{R} \& \mathrm{D}$ and systems analysis services in remote sensing, surveillance, monitoring and tracking - (SIC Code - $\underline{8700})$

- $\underline{\mathrm{MAN}}-\underline{\mathrm{EP}}-\underline{\mathrm{PB}} \ldots . .$. Plasma etching reactors - (SIC Code - $\underline{3559})$

- MAN - MA ...... Plasma etching reactors - (SIC Code - 3559) 
Códe - 8731)

- $\underline{\text { PHO }}$ - LA - $\underline{\text { SS }}$....... Laser probes - (SIC Code - $\underline{3699)}$

$\circ \underline{\mathrm{PHO}}-\underline{\mathrm{SV}}-\underline{\mathrm{R}}$....... Laboratory and theoretical research services in optics and electro-optics - (SIC Code 8731)

- $\underline{\mathrm{SOF}}-\underline{\mathrm{GO}}-\underline{\mathrm{M}}$....... Advanced spectral signatures computer models software - (SIC Code - $\underline{7372}$ )

- $\underline{\mathrm{SOF}}-\underline{\mathrm{PD}}-\underline{\mathrm{F}} \ldots .$. . Optical computing and optical signal processing algorithms software $-($ SIC Code $-\underline{7372}$ )

- $\underline{\text { TAM }}$ - $\underline{\mathrm{AN}}-\underline{\mathrm{E}}$...... Spectral monitors - (SIC Code - $\underline{3825}$ )

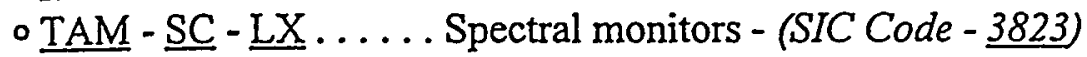

- TEL - BR - TEIPS ...... Point source and remote monitoring systems - (SIC Code - 3663)

- $\underline{T E L}$ - $\underline{\text { I }}-\underline{\text { PO }}$...... Optical computing and optical signal processing circuits - (SIC Code - $\underline{3669)}$ 


\section{Excel Quantronix Corp.}

- SHC: Control Laser International Corp.

\section{Address:}

45 Adams Ave.

Hauppauge. NY 11787

Tel: (516) 273-6900; (800) 289-7707

Fax: (516) 273-6958

\section{Internet:}

Explore: Resource Pages

\section{Status:}

Operating unit of a public U.S. company (Ticker symbol: XTLC). This operating unit formed in 1967. Ultimate parent is Excel Technology. Inc.

Size:

- Empl - 50 - 99 employees; the employee growth rate for the year prior to interview is unknown.

- Sales - $\$ 10 \mathrm{~m}-\$ 25 \mathrm{~m}$ annual sales; $10 \%$ to $25 \%$ of sales are from international business.

\section{Execs:}

- Antoine Dominick, President (C.E.O., Finance)

- Steve Hoffman, Purchasing Manager (Purchasing)

- Ms. Carmela Dimiao, M.I.S. Manager (M.I.S.)

\section{Primary Industry: Photonics (PHO)}

Primary SIC Code: 3699

Manufacturer of Nd: YAG lasers and systems, spectroscopy laser systems and indentification marking laser systems. Products are used in industrial, scientific and semiconductor applications. This company was capitalized by private investment, venture capital and corporate investment.

\section{Product Codes:}

- $\underline{\mathrm{AUT}}-\underline{\mathrm{MC}}-\underline{\mathrm{D}}$...... Identification marking laser systems - (SIC Code - $\underline{3625}$ )

- $\underline{\text { HO }}$ - LA - LSN ...... Nd:YAG lasers and systems - (SIC Code - 3699)

- $\underline{\mathrm{PHO}}-\underline{\mathrm{LA}}-\underline{\mathrm{SS}}$...... . Spectroscopy laser systems - (SIC Code - $\underline{3699)}$ 


\section{MetroLaser, Inc.}

\section{Address:}

18006 Skypark Cir., Suite 108

Irvine. CA $\underline{92614}$

Tel: (714) 553-0688

Fax: (714) 553-0495

Internet:

Explore: Resource Pages

HomePage: http://www.pages.prodigy.com

e-mail: metro@deltanet.com

\section{Status:}

Independent private U.S. company. Minority owned. Formed in 1988.

Government Contractor: Prime \& Sub-contract

Size:

- Empl - 33 employees; $10 \%$ employment growth in the year prior to interview; $6 \%$ employment growth projected for the next fiscal year.

- Sales - $\$ 5 \mathrm{~m}-\$ 10$ annual sales; under $2.5 \%$ of sales are from international business.

Execs:

- Dr. Cecil Hess Ph.D., President (C.E.O., Finance)

- Dr. James Trollinger Ph.D., Vice President (Sales, R\&D)

Primary Industry: Photonics (PHO)

Primary SIC Code: 3699

Provider of research and development services specializing in holography and spectroscopy laser systems. Services are provided primarily to the government. This company was capitalized by private investment.

\section{Product Codes:}

- $\underline{\mathrm{PHO}}$ - $\underline{\mathrm{LA}}$ - $\underline{\mathrm{SH}}$...... Holography laser systems research and development - (SIC Code - $\underline{3699}$ )

$\circ \underline{\text { PHO }}-\underline{\text { LA }}-\underline{\text { SS }}$...... Spectroscopy laser systems research and development - (SIC Code $-\underline{3699}$ )

- $\underline{\mathrm{PHO}}-\underline{\mathrm{SV}}-\underline{\mathrm{R}} . . . .$. . Photonics research and development services - (SIC Code $-\underline{8731}$ ) 


\section{Photon Technology International, Inc.}

- AKA: PTI

Address:

1 Deerpark Dr., Suite F

Monmouth Junction. NJ 08852

Tel: (908) 329-0910

Fax: (908) 329-9069

\section{Internet:}

Explore: Resource Pages

\section{Status:}

Public U.S. company (Ticker symbol: PHON). Formed in 1983.

Government Contractor: Prime \& Sub-contract

Size:

- Empl - 62 employees; $9 \%$ employment reduction in the year prior to interview; $4 \%$ employment growth projected for the next fiscal year.

- Sales - Annual sales $=\$ 9.1$ million; over $25 \%$ of sales are from international business.

\section{Execs:}

- Charles G. Marianik, COB/CEO/President (Chairman, C.E.O., Corporate Dev., Strategic Planning)

- Ron Kovach, Vice President/Secretary (R\&D)

- William D. Looney, Vice President/Controller/Treasurer (Finance, Administration, Personnel, Purchasing)

- G. Reicher, Director of International Marketing (Int'l Sales \& Mktg.)

- Jerry DeVries, Engineering Manager (Engineering)

- M. Beattie, Manufacturing Manager (Manufacturing)

- Cary Davies, Domestic Sales Manager (Sales)

- David Smith, Marketing Manager (Marketing)

Primary Industry: Photonics (PHO)

Primary SIC Code: 3679

Manufacturer of lamps and lamp housings, ICCD cameras, optical choppers, monochromators, nitrogen and dye lasers, laser dyes, fluorescence spectrophotometer systems for physical chemistry applications, sample compartments, photomultipliers and photomultiplier power supplies, stepping motor drives, light source power supplies, fluorescence lifetime systems and fluorescence spectrophotometers. Products are sold to the biochemistry "and biotechnology industries. This company was capitalized by private investment and venture capital.

\section{Product Codes:}

:

- $\underline{\mathrm{PHO}}$ - $\underline{\mathrm{CA}}-\underline{\mathrm{SC}}$...... ICCD cameras - (SIC Code - $\underline{3861})$

- $\underline{\mathrm{PHO}}-\underline{\mathrm{LA}}-\underline{\mathrm{CD}} \ldots .$. Laser dyes - (SIC Code - $\underline{3699)}$

- $\underline{\mathrm{PHO}}-\underline{\mathrm{LA}}-\underline{\mathrm{LGZ}}$..... . Nitrogen lasers - (SIC Code - $\underline{\mathrm{3699}})$

- $\underline{\mathrm{PHO}}-\underline{\mathrm{LA}}-\underline{\mathrm{LL}} \ldots .$. ... Dye lasers - (SIC Code - $\underline{3699)}$

$\therefore \quad 0 \underline{\mathrm{PHO}}-\underline{\mathrm{LA}}-\underline{\mathrm{SS}}$..... Fluorescence spectroscopy systems - (SIC Code - 3699)

- $\underline{\mathrm{PHO}}-\underline{\mathrm{OE}}-\underline{\mathrm{LA}} \ldots .$. . Arc lamps - (SIC Code - $\underline{3640})$

. $\quad \underline{\mathrm{PHO}}-\underline{\mathrm{OE}}-\underline{\mathrm{LS}} \ldots .$. Stroboscopic lamps - (SIC Code $-\underline{3640})$





- $\underline{\mathrm{PHO}}-\underline{\mathrm{OE}}-\underline{\mathrm{LU}}$...... Ultraviolet lamps - (SIC Code - $\underline{\mathrm{3640}}$ )
- $\underline{\mathrm{PHO}}-\underline{\mathrm{OE}}-\underline{\mathrm{LX}}$....... Xenon lamps - (SIC Code - $\underline{3640)}$
- $\underline{P H O}-\underline{\mathrm{OE}}-\underline{\text { IY }} \ldots . .$. . Light source power supplies - (SIC Code - $\underline{3679)}$
- PHO - OE - LY..... A Arc lamp power supplies - (SIC Code - 3679)
- $\underline{\text { PHO }}$ - $\underline{\mathrm{OE}}-\underline{\mathrm{P}}$....... Photomultipliers - (SIC Code - $\underline{3671)}$
- $\mathrm{PHO}$ - $\mathrm{OP}$ - EZ ...... Sample compartments - (SIC Code - 3827)
$\circ \underline{\mathrm{PHO}}-\underline{\mathrm{OP}}-\underline{\mathbb{N}} \ldots .$. Monochromaters and accessories - (SIC Code - $\underline{3826})$
- $\underline{\mathrm{PHO}}-\underline{\mathrm{OP}}-\underline{\mathrm{IR}}$....... Microscope photometers - (SIC Code - $\underline{3826)}$
- PHO - OP - PE ...... Optical choppers - (SIC Code - 3827)
- $\underline{\mathrm{SUB}}-\underline{\mathrm{ES}}-\underline{\mathrm{PQ}} \ldots$.... . Photomultiplier power supplies - (SIC Code $-\underline{3679}$ )
- $\underline{T A M}$ - $\underline{\text { PV }}-\underline{E}$....... Stepping motor drives - (SIC Code - 3823) -
- $\underline{\mathrm{TAM}}$ - $\underline{\mathrm{SC}}-\underline{\mathrm{IR}}$....... Fluorescence spectrophotometer systems - (SIC Code - 3826) 


\section{FIBER OPTIC SENSORS}




\section{Ciencia, Inc.}

- FKA: Tech Quest, Inc.

\section{Address:}

111 Roberts St., Suite K

East Hartford $\underline{\text { CT }} \underline{06108}$

Tel: (860) 528-9737

Fax: (860) 528-5658

\section{Internet:}

Explore: Resource Pages

e-mail:74503.126@compuserve.com

Status:

Independent private U.S. company. Minority owned. Formed in 1989.

Government Contractor: Prime \& Sub-contract

Size:

- Empl - 13 employees; $30 \%$ employment growth in the year prior to interview; no employment change projected for the next fiscal year.

- Sales - Estimated annual sales $=\$ 1.5$ million; no international sales.

\section{Execs:}

- Salvador M. Fernandez, President (C.E.O.)

- Arturo O. Pilar, Vice President (Sales, Marketing)

- Ms. Sue Wightman, Comptroller (Finance)

- Dr. Emie Guignon, Director of Engineering (R\&D, Engineering)

- Ralph Levy, Director of New Product Development (Manufacturing)

- Gary Bennett, Vice President

Primary Industry: Photonics (PHO)

Prifnary SIC Code: 3827

Developer of optical diagnostics instrumentation for biomedical, environmental and process analysis markets.

Products include tunable optical filters, holographic filters, fiber-optic based sensors for on-line real-time chemical analysis, hyperspectral imaging polarimeters for remote sensing, phase fluorometry instrumentation, Raman spectroscopy instrumentation for optical biopsy of tissues, biosensors and a fluorescence-based cell analysis system. The company also provides contract research and development services to the government and corporate clients. Provider of industrial hygiene services. Products and services are sold to multiple industries.

\section{Product Codes:}

- $\underline{B I O}$ - EL - $\underline{B}$....... Biosensors - (SIC Code - 3826 )

- $\underline{B I O}$ - EL - $\underline{\text { S } . . . . . . ~ F l o u r e s c e n c e-b a s e d ~ c e l l ~ a n a l y s i s ~ s y s t e m s ~-~(S I C ~ C o d e ~}-$ 3826)

- $\underline{\mathrm{MED}}$ - $\underline{\mathrm{SV}}-\underline{\mathrm{R}}$....... Biomedical research and development services - (SIC Code - $\underline{8731}$ )

- $\underline{\mathrm{PHO}}-\underline{\mathrm{AO}}-\underline{\mathrm{Z}}$...... . Acousto-optic tunable filters - (SIC Code $-\underline{3827}$ )

- $\underline{\mathrm{PHO}}-\underline{\mathrm{OP}}-\mathrm{FZ} \ldots .$. . Acousto-optic tunable filters - (SIC Code - $\underline{\text { 3827) }}$

- $\underline{\mathrm{PHO}}-\underline{\mathrm{OP}}-\underline{\mathrm{H}}$...... Holographic filters - (SIC Code - $\underline{3827}$ )

$\circ \underline{\mathrm{PHO}}-\underline{\mathrm{SV}}-\underline{\mathrm{R}} . . . .$. . Photo-optic research and development services - (SIC Code $-\underline{8731}$ )

- $\underline{\mathrm{SUB}}-\underline{\mathrm{SV}}-\underline{\mathrm{R}} . . .$. . Electronics research and development services - (SIC Code - $\underline{8731}$ )

- $\underline{\text { TAM }}$ - $\underline{\mathrm{AN}}-\underline{\mathrm{C}} . . .$. . On-line real-time chemical analysis systems - (SIC Code $-\underline{3826})$ 
- $\underline{\text { TAM }}-\underline{\mathrm{ME}}-\underline{\mathrm{P}}$...... Hyperspectral imaging polarimeters - (SIC Code - $\underline{3829}$ )

- IAM - $\underline{\text { SC }}-\underline{\text { IH }}$...... . Spectroscopy instrumentation - (SIC Code - $\underline{3826)}$

- $\underline{\text { TAM }}-\underline{\mathrm{SC}}-\underline{\mathrm{IZ}}$...... Phase flourometry instrumentation - (SIC Code - $\underline{3829}$ ) 


\section{Detection Limit, Inc.}

- FKA: Detection Limit Technology

\section{Address:}

555 General Brees Rd.

Laramie, WY 82070

Tel: (307) 742-0555

Fax: (307) 742-0168

\section{Internet:}

Explore: Resource Pages

e-mail: dlt@uwyo.edu

Status:

Independent private U.S. company. Formed in 1991.

Government Contractor: Prime \& Sub-contract

\section{Size:}

- Empl - 6 employees; no employment change in the year prior to interview; no employment change projected for the next fiscal year.

- Sales - Annual sales $=\$ .80$ million; no international sales .

\section{Execs:}

- Dr. Christian Schoen, President (C.E.O., Sales, R\&D, M.I.S.)

- Eugene Watson, Vice President (Marketing)

Primary Industry: Test \& Measurement (TAM)

Primary SIC Code: 3825

Manufacturer of fiber optic environment sensors used for containment detection. Products are sold to multiple industries. This company was capitalized by private investment.

\section{Product Codes:}

- TAM - DE ...... Containment detectors - (SIC Code - $\underline{3820)}$

- $\underline{\mathrm{TAM}}-\underline{\mathrm{ME}}-\underline{\mathrm{P}}$...... . Fiber optic environment sensors - (SIC Code - $\underline{3829})$ 


\section{Lightsense Corp.}

Address:

955 South Virginia St., Suite 116

Reno. NV 89502

Tel: (702) 348-0899

Fax: (702) 324-1399

Internet:

Explore: Resource Pages

e-mail: lightsense@lamg.com

Status:

Independent private U.S. company. Formed in 1986.

Size:

- Empl - Under 10 employees; the employee growth rate for the year prior to interview is unknown.

- Sales - Under $\$ 1 \mathrm{~m}$ annual sales; $10 \%$ to $25 \%$ of sales are from international business.

Execs:

- Lawrence S. Ring, President (C.E.O., Sales, Marketing, Finance, Manufacturing, Engineering)

Primary Industry: Test \& Measurement (TAM)

Primary SIC Code: 3825

Manufacturer of fiber optic chemical sensors for medical and industrial uses. Products are sold to the medical and biotechnology industries. This company was capitalized by private investment.

\section{Product Codes:}

- $\underline{\text { BIO }}$ - EL - $\underline{\text { B }} . . .$. . Fiber optic chemical sensors - (SIC Code - $\underline{3826}$ )

- TAM - AN - ….... Fiber optic chemical sensors - (SIC Code - 3826)

- TAM - ME - MZ ...... Fiber optic chemical sensors - (SIC Code - 3829)

- IAM - ME - P..... Fiber optic chemical sensors - (SIC Code - 3829) 


\section{Ocean Optics, Inc.}

\section{Address:}

1237 Lady Marion Ln.

Dunedin, FL 34698

Tel: (813) 733-2447

Fax: (813) 733-3962

\section{Internet:}

Explore: Resource Pages

HomePage: http://ourworld.compuserve.com/homepages/ocean-optics

e-mail: 75461.2007@compuserve.com

\section{Status:}

Independent private U.S. company. Formed in 1989.

Size:

- Empl-14 employees; $16 \%$ employment growth in the year prior to interview; $7 \%$ employment growth projected for the next fiscal year.

- Sales - Annual sales $=\$ 2.4$ million; $10 \%$ to $25 \%$ of sales are from international business .

\section{Execs:}

- Mike Morris, President (C.E.O.)

- Lee Bean, VP of Sales and Marketing (Sales, Marketing)

- Scott Bangert, VP of Manufacturing (Manufacturing)

- Ms. Lori Detwiler, Controller (Finance)

- Dr. Roy Walters, Director of Research (R\&D).

- Rob Morris, Marketing Managẹr

Primary Industry: Test \& Measurement (TAM)

Primary SIC Code: 3825

Manufacturer of miniature fiber optic spectrometers, NIR fiber optic spectrometers, fiber optic probes and accessories, ultraviolet light sources, fiber optic $\mathrm{pH}$ sensors and fiber optic toxic metal sensors. Products are used primarily for color analysis, chemical composition, light measurement and particle size analysis applications. Products are sold to multiple industries. This company was capitalized by private investment and venture capital.

\section{Product Codes:}

- $\underline{\text { AUT }}$ - $\underline{\mathrm{AT}}-\underline{\mathrm{A}}$........ Fiber optic probes - (SIC Code $-\underline{3829}$ )

$\circ \underline{\mathrm{PHO}}-\underline{\mathrm{FO}}-\underline{\mathrm{PZ}}$....... Fiber optic probes/accessories - (SIC Code $-\underline{\text { 3827) }}$

- $\underline{\text { PHO }}-\underline{\mathrm{OE}}-\underline{\mathrm{LU}}$...... . Ultraviolet light sources - (SIC Code - $\underline{3640)}$

- TAM - ME - MO ...... Fiber optic pH sensors - (SIC Code - $\underline{3826)}$

- $\underline{\text { TAM }}-\underline{\mathrm{ME}}-\underline{\mathrm{P}}$....... Fiber optic toxic metal sensors - (SIC Code - 3829)

- $\underline{\text { TAM }}$ - $\underline{\text { ME }}$ - $\underline{\mathrm{P}}$...... . Fiber optic pH sensors - (SIC Code $-\underline{3829)}$

- $\underline{\text { TAM }}$ - $\underline{\text { SC }}$ - ISZ ...... NIR fiber optic spectrometers - (SIC Code $-\underline{3829)}$

- $\underline{\text { IAM }}$ - $\underline{\text { SC }}$ - ISZ ..... . Miniature fiber optic spectrometers - (SIC Code - $\underline{3829}$ ) 


\section{Orbital Sciences Corp. / Sensor Systems Division}

-FKA: Orbital Sciences Corp. / Pomona Operation

Address:

2771 North Garey Ave., PO Box 2801

Pomona CA 91769

Tel: (909) 593-3581

Fax: (909) 596-2301

\section{Internet:}

Explore: Resource Pages

HomePage: http://www.orbital.com

\section{Status:}

Operating unit of a public U.S. company (Ticker symbol: ORBI). This operating unit formed in 1957. Ultimate parent is Orbital Sciences Corp.

Government Contractor: Prime \& Sub-contract

Size:

- Empl- 175 employees; $6 \%$ employment growth in the year prior to interview; $8 \%$ employment growth projected for the next fiscal year.

- Sales - Annual sales $=\$ 22$ million; under $2.5 \%$ of sales are from international business.

\section{Execs:}
- Robert Hertel, General Manager/Senior Vice President (C.E.O., Finance, R\&D)
- Ulli Hsrtmann, Director of Engineering (Engineering)
- Steve Colosimo, M.I.S. Manager (M.I.S.)
- D. McDenmott, Purchasing Manager (Purchasing)

Primary Industry: Environmental (ENV)

Primary SIC Code: 3823

Manufacturer of a wide variety of optical equipment, analytical instruments and high-reliability space application electronics. Products and services are sold to multiple industries.

\section{Product Codes:}

- $\underline{\mathrm{DEF}}-\underline{\mathrm{EW}}-\underline{\mathrm{CO}} \ldots .$. Optical intelligence receivers - (SIC Code $-\underline{3812}$ )

- $\underline{\mathrm{DEF}}-\underline{\mathrm{EW}}-\underline{\mathrm{CO}} \ldots .$. Optical countermeasure systems - (SIC Code - 3812)

- $\underline{\mathrm{DEF}}-\underline{\mathrm{EW}}-\underline{\mathrm{D}} \ldots \mathrm{.} . \mathrm{.}$. Strategic defense initiative systems - (SIC Code - 3812$)$

- $\underline{\mathrm{DEF}}-\underline{\mathrm{ST}} \ldots . . \mathrm{Star}$ trackers/sensors - (SIC Code - 3812)

- $\underline{\mathrm{DEF}}-\underline{\mathrm{ST}} \ldots . .$. Reconnaissance systems - (SIC Code - 3812)

- $\underline{\text { ENR }}-\underline{\mathrm{EP}}-\underline{\mathrm{U}}$..... . Uninterruptible power supplies - (SIC Code - $\underline{3679)}$

- $\underline{E N V}-\underline{A N}-\underline{G}$..... Industrial atmosphere (gas) analyzers - (SIC Code - 3823)

- ENV - $\underline{A N}-\underline{G} \ldots .$. Industrial gas analyzers - (SIC Code - 3823)

- $\underline{\text { MED }}$ - $\underline{\mathrm{DG}}-\underline{\mathrm{D}}$...... Microdensitometers - (SIC Code - $\underline{3841})$

- $\underline{\mathrm{PHO}}-\underline{\mathrm{CA}}-\underline{\mathrm{M}}$.....16 mm and $35 \mathrm{~mm}$ gun/aerial panoramic cameras - (SIC Code - $\underline{3861})$

- $\underline{\mathrm{PHO}}-\underline{\mathrm{LA}}-\underline{\mathrm{AD}} \ldots . .$. Optical detectors - (SIC Code - 3827)

- $\underline{\mathrm{PHO}}-\underline{\mathrm{LA}}-\underline{\mathrm{AD}} \ldots .$. ... Laser detectors - (SIC Code - 3827)

$\therefore \quad$ PHO $-\underline{\mathrm{OE}}-\underline{\mathrm{D}} \ldots . .$. Infrared detectors - (SIC Code - 3679$)$




o $\underline{\mathrm{PHO}}$ - $\underline{\mathrm{OP}}$ - IVT ...... Infrared telescopes - (SIC Code - $\underline{3827)}$

- $\underline{\text { TAM }}-\underline{\mathrm{AN}}-\underline{\mathrm{C}}$...... Industrial liquid analyzers - (SIC Code $-\underline{3826}$ )

- TAM - ME - MD ...... Microdensitometers - (SIC Code - $\underline{3826)}$

- TAM - ME - P..... Optical sensors - (SIC Code - 3829$)$

- IAM - $\underline{\text { SC }}-\underline{\text { ISM }}$..... . Mass spectrometers - (SIC Code - 3826)

- IAM - SC - ISZ ...... Optical spectrometers - (SIC Code - 3829)

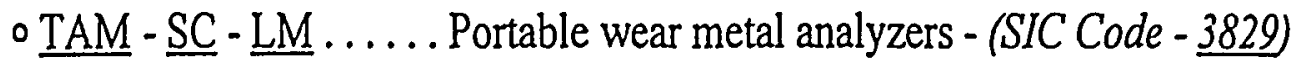

- TAM - SV ...... Optical testing services - (SIC Code - 8734)

- TEL - BR - TEIPS ....... Remote sensing systems - (SIC Code - 3663) 


\section{Sweeney Aquametrics}

\section{Address:}

PO Box 3369

Stony Creek, CT 0.5405

Tel: (203) 488-9752; (800) 647-3971

Fax: (203) 488-4099

\section{Internet:}

Explore: Resource Pages

\section{Status:}

Independent private U.S. company. Formed in 1986.

Government Contractor: Prime

Size:

- Empl - 3 employees; no employment change in the year prior to interview; $66 \%$ employment growth projected for the next fiscal year.

- Sales - Annual sales $=\$ .75$ million; $2.5 \%$ to $10 \%$ of sales are from international business.

\section{Execs:}

- John W. Sweeney, President (C.E.O., Sales, R\&D, Finance, M.I.S.)

- Fred Maurer, Manufacturing Manager (Manufacturing)

Primary Industry: Environmental (ENV)

Primary SIC.Code: 3823

Manufacturer of water analysis instruments for water quality measurement and total dissolved pressure sensors. Products are sold to fish hatcheries, environmental monitoring organizations and businesses such as hydro-electric companies. This company was capitalized by private investment.

\section{Product Codes:}

- $\underline{\text { ENV }}-\underline{\mathrm{AN}}-\underline{\mathrm{W}} \ldots . .$. Water analysis instruments - (SIC Code - $\underline{3823}$ )

- $\underline{\mathrm{TAM}}$ - $\underline{\mathrm{ME}}-\underline{\mathrm{P}}$...... . Pressure sensors - (SIC Code $-\underline{3829}$ ) 


\section{UOP / Guided Wave Process Analytical Systems}

\section{Address:}

5190 Golden Foothill Pkwy.

El Dorado Hills, CA 95762

Tel: (916) 939-4300

Fax: (916) 939-4307

\section{Internet:}

Explore: $\underline{\text { Resource Pages }}$

\section{Status:}

Operating unit of a public U.S. company (Ticker symbol: UK). This operating unit formed in 1983. Ultimate parent is Union Carbide Corp.

Size:

- Empl-2,500 - 4,999 employees; the employee growth rate for the year prior to interview is unknown.

- Sales - Ultimate parent had annual sales $=\$ 6,100$ million; over $25 \%$ of sales are from international business.

\section{Execs:}

- Michael Winfield, President (Chairman)

- Keith Aspray, Director (C.E.O.)

- Dr. Terry Todd, Manager of Research and Development (R\&D)

- Marvin Cook, Manager of Sales and Marketing (Sales, Marketing)

- William Grooms, Manager of Manufacturing (Manufacturing)

- Brian Mindel, Sales Manager (Int'l Sales \& Mktg.)

- Ms. Elizabeth Davis, Buyer (Purchasing)

Prinary Industry: Test \& Measurement (TAM;

Primary SIC Code: 3820

Manufacturer of NIR/infrared analyzers, probes, spectrum analyzers, spectrophotometers, rapid scanning spectrometers, fiber optic cable and other accessories used with laboratory, process and turnkey systems. This company is a joint venture between Union Carbide Corp. and AlliedSignal Inc. Products are sold to the chemical process and refinery industries. This company was capitalized by corporate investment.

\section{Product Codes:}

- $\underline{\mathrm{PHO}}-\underline{\mathrm{FO}}-\underline{\mathrm{CZ}} \ldots . .$. Fiber optic cables $-($ SIC Code $-\underline{3357})$

- TAM - AN - E..... Spectrum analyzers - (SIC Code $-\underline{3825)}$

- TAM - $\underline{\text { AN }}$ - OI ...... NIR/infrared analyzers - (SIC Code - $\underline{3823)}$

- $\underline{\text { TAM }}$ - $\underline{\text { SC }}-\underline{\text { IR }}$...... Spectrophotometers - (SIC Code - $\underline{\text { 3826) }}$

- $\underline{\text { TAM }}-\underline{\underline{S C}}-\underline{\underline{\text { ISZ }}}$...... Rapid scanning spectrometers - (SIC Code $-\underline{3829})$

- $\underline{T A M}-\underline{S C}-\underline{S B}$...... Fiber optic probes - (SIC Code - $\underline{3826)}$ 


\section{ULTRA-VIOLET OPEN PATH SENSORS}




\section{Thermo Environmental Instruments, Inc.}

o PMW: Wedding \& Associates, Inc.

\section{Address:}

8 West Forge Pkwy.

Franklin, MA 02038

Tel: (508) 520-0430

Fax: (508) 520-1460

\section{Internet:}

Explore: Resource Pages

\section{Status:}

Operating unit of a public U.S. company (Ticker symbol: TMO). This operating unit formed in 1970. Ultimate parent is Thermo Electron Corp.

\section{Government Contractor: Prime}

Size:

- Empl- 205 employees; no employment change in the year prior to interview.

- Sales - Estimated annual sales $=\$ 25$ million; over $25 \%$ of sales are from international business.

\section{Execs:}

- Denis Helm, President (C.E.O., Finance, R\&D)

- C.J. Gabriel, VP of Marketing (Sales, Marketing)

- Mike Nemergut, Internationsal Marketing Manager (Int'l Sales \& Mktg.)

Primary Industry: Environmental (ENV)

Primary SIC Code: 3823

Manufacturer of pollution analysis and measurement equipment. Products include gas, stack gas and ambient air analyzers, air quality monitoring devices and portable and continuous hydrocarbon monitors. Products are sold to multiple industries.

\section{Product Codes:}

- $\underline{E N V}$ - $\underline{\mathrm{AN}}-\underline{\mathrm{A}}$....... Ambient air analyzers - (SIC Code - 3823)

- ENV - $\underline{A N}-\underline{G}$...... Gas analyzers - (SIC Code $-\underline{3823)}$

- ENV - $\underline{\text { AN }}-\underline{G}$...... Stack gas analyzers - (SIC Code - $\underline{3823}$ )

- ENV - $\underline{\text { CP }}-\underline{A}$....... Air quality monitoring devices - (SIC Code $-\underline{3564)}$

- ENV - $\underline{\text { P }}-\underline{A}$....... Portable and continuous hydrocarbon monitors - (SIC Code - 3564) 


\section{LIDAR SYSTEMS}

Fi 


\section{B.E. Meyers and Co., Inc.}

- AKA: Meyers and Co., Inc.

\section{Address:}

17525 Northeast 67 th $\mathrm{Ct}$.

Redmond. WA 98052

Tel: (206) 881-6648; (800) 327-5648

Fax: (206) $867-1759$

\section{Internet:}

Explore: Resource Pages

\section{Status:}

Independent private U.S. company. Formed in 1976.

\section{Government Contractor: Prime \& Sub-coniract}

\section{Size:}

- Empl-25 employees; no employment change in the year prior to interview; no employment change projected - for the next fiscal year.

- Sales - Estimated annual sales $=\$ 4.3$ million; no international sales.

\section{Execs:}

- Brad Meyers, President (C.E.O.)

- Roger Kreitzberg, VP of Marketing (Sales, Marketing, Int'l Sales \& Mktg.)

- Ms. Nancy Meyers, Secretary/Treasurer (Finance)

- Ms. Gwynn Wilkinson, Controller (Purchasing, Personnel)

- Christopher Tott, Production Manager (Manufacturing, R\&D)

Primary Industry: Telecommunications (TEL)

Primary SIC Code: 3663

Manufacturer of Dark Invader ${ }^{\mathrm{TM}}$, microwave television and data transmission systems; night vision systems; day/night TV cameras; day/night surveillance units; and long-range infrared laser illumination systems. Products are sold to the military, police/security agencies, research companies and NASA.

\section{Product Codes:}

- $\underline{\mathrm{PHO}}-\underline{\mathrm{LA}}-\underline{\mathrm{SR}} \ldots . .$. Ranging and scanning laser systems - (SIC Code $-\underline{3699})$

- PHO - OE - LR ...... Infrared laser illumination - (SIC Code - 3640)

- TEL - $\underline{\text { AV }}-\underline{Z}$...... Image enhancing, ultra low-light-level video systems - (SIC Code - 3669)

- TEL - BR - TEIl ...... Forward looking infrared radar imaging systems - Dark Invader ${ }^{\mathrm{TM}}$ - (SIC Code 3663)

- TEL - BR - TEIPD ...... Digital image processors - (SIC Code - 3663)

- TEL - BR - TEIPE ...... Image enhancing equipment - (SIC Code - 3663)

- TEL - BR - TEIPS ...... Remote sensing equipment - (SIC Code - 3663)

- TEL - BR - TET ...... Microwave television transmission systems - (SIC Code - 3663)

- $\underline{T E L}-\underline{\text { SM }}$ - $\underline{\text { ME }}$....... Microwave data transmission systems - (SIC Code - $\underline{3663}$ ) 


\section{Computer Genetics Corp.}

\section{Address:}

34 Woodcrest Rd.

Boxford. MA 01921

Tel: (508) 887-8389

Fax: (508) 887-9088

Internet:

Explore: $\underline{\text { Resource Pages }}$

Status:

Independent private U.S. company. Formed in 1974.

Government Contractor: Prime \& Sub-contract

Size:

- Empl - 6 employees; no employment change in the year prior to interview; no employment change projected for the next fiscal year.

- Sales - Annual sales $=\$ 1.0$ million; no international sales.

\section{Execs:}

- Bernard Caputo, President (C.E.O., Finance, Sales, R\&D)

- Ms. Agness Hanne, Office Manager (Administration)

Primary Industry: Environmental (ENV)

Primary SIC Code: 8700

Provider of light detection and ranging and opacity measurement services. Also a researcher and developer of gas monitoring systems and test and measurement equipment for industrial or aerospace markets and smokestack and toxic and hazardous gas monitoring services. Services are offered to the manufacturing, utility and government markets. Products are sold to the petroleum industry, manufacturers and public utilities.

\section{Preduct Codes:}

- $\underline{\mathrm{ENV}}-\underline{\mathrm{SV}}-\underline{\mathrm{R}}$...... Gas monitoring research and development services - (SIC Code - $\underline{8731)}$

- IAM - SV ...... Light detection and ranging services - (SIC Code - 8734)

- $\underline{\text { TAM }}$ - SV ...... Opacity measurement services - (SIC Code - 8734)

- $\underline{\text { TAM }}$ - $\underline{\text { SV }} . . . .$. Test \& measurement research development services - (SIC Code - 8734) 


\section{System Specialists, Inc.}

\section{Address:}

4001 North Runway Dr., Suite 151

Tucson. AZ 85705

Tel: (520) 292-9644

Fax: (520) 292-9621

\section{Internet:}

Explore: Resource Pages

-HomePage: http://www.spiderhsv.com/ssi/

e-mail:wpoteet@aol.com

Status:

Independent private U.S. company. Formed in 1972.

Government Contractor: Prime \& Sub-contract

Size:

- Empl - 6 employees; no employment change in the year prior to interview; no employment change projected for the next fiscal year.

- Sales - Estimated annual sales $=\$ .70$ million; $2.5 \%$ to $10 \%$ of sales are from international business.

\section{Execs:}

- Harold Cauthen, President (C.E.O., Finance)

- Wade M. Poteet, VP of Research and Development (R\&D, Sales)

- Kenneth Blaisdell, Director of Engineering (Engineering)

- John Holcomb, Optical Scientist (M.I.S.)

- David Cummings, Foreman of Manufacturing (Manufacturing)

Primary Industry: Photonics (PHO)

Primary SIC Code: 3827

Manufacturer of LIDAR environmental sensing systems and ultrahigh precision laser positioning sensors and optical telescopes and components. Provider of electronics, cryogenics, mechanical electro-optics and infrared systems design and manufacturing services. Products include infrared astronomy systems. Products and services are provided to commercial companies, the military, NASA, universities and other government agencies.

\section{Product Codes:}

- $\underline{\mathrm{PHO}}-\underline{\mathrm{CA}}-\underline{\mathrm{M}}$....... Infrared cameras - (SIC Code - $\underline{3861}$ )

- $\underline{\mathrm{PHO}}-\underline{\mathrm{OP}}-\underline{\mathrm{IA}}$...... LIDAR astronomical instruments - (SIC Code - $\underline{\text { 3827) }}$

$\circ \underline{\mathrm{PHO}}-\underline{\mathrm{OP}}-\underline{\text { IVT }}$....... Optical telescopes and components - (SIC Code - $\underline{3827)}$

- $\underline{\mathrm{PHO}}-\underline{\mathrm{SV}}-\underline{\mathrm{D}}$....... Electro-optics design services - (SIC Code $-\underline{\text { 8711) }}$

- $\underline{\mathrm{PHO}}-\underline{\mathrm{SV}}-\underline{\mathrm{M}}$....... Mechanical electro-optics manufacturing services - (SIC Code - 8711$)=$

- $\underline{\mathrm{PHO}}-\underline{\mathrm{SV}}-\underline{\mathrm{R}} . . . .$. Mechanical electro-optics research and development services - (SIC Code $-\underline{8731}$ )

- $\underline{\mathrm{SUB}}-\underline{\mathrm{EM}}-\underline{\mathrm{D}}$....... Cryogenic equipment - (SIC Code - $\underline{3585}$ )

- $\underline{\mathrm{SUB}}-\underline{\mathrm{SV}}-\underline{\mathrm{M}}$...... Electronics manufacturing services - (SIC Code $-\underline{8711})^{-}$

- $\underline{\mathrm{SUB}}-\underline{\mathrm{SV}}-\underline{\mathrm{R}} \ldots . .$. Electronics research and development services - (SIC Code $-\underline{8731}$ )

- $\underline{\mathrm{TAM}}$ - ME $-\underline{\mathrm{P}}$...... . Laser positioning sensors - (SIC Code - $\underline{3829})$ 


\section{THERMAL IMAGING OR SENSING}




\section{Daedalus Enterprises, Inc.}

\section{Address:}

300 Parkland Plaza, PO Box 1869

Ann Arbor, MI 48106

Tel: (313) 769-5649

Fax: (313) 769-0429

\section{Internet:}

Explore: Resource Pages

\section{Status:}

Public U.S. company (Ticker symbol: DDEI). Formed in 1968.

Government Contractor: Prime \& Sub-contract

Size:

- Empl - 17 employees; $35 \%$ employment reduction in the year prior to interview; no employment change projected for the next fiscal year.

- Sales - Annual sales $=\$ 2.5$ million; over $25 \%$ of sales are from international business.

\section{Execs:}

- John Sanders, Chairman of the Board (Chairman)

- Thomas Ory, President/CEO (C.E.O., Personnel, Sales, Marketing)

- Charles G. Stanich, Vice President (R\&D, Manufacturing)

- Ms. Jane E. Barrett, Treasurer (Finance)

- Robert England, Purchasing Agent (Purchasing)

Primary Industry: Test \& Measurement (TAM)

\section{Primary SIC Code: 3825}

Manufacturer of airborne remote-sensing systems. The infrared, heat-seeking equipment is used to protect coastlines against hazardous waste spills, to detect hot spots in forest fires, in mineral exploration, for pipeline 'mapping, for geological studies, in mining applications and for other environmental applications. This company was capitalized by private investment and corporate investment.

\section{Product Codes:}

- ENR - MI ...... Heat-seeking equipment - (SIC Code - $\underline{3532)}$

- ENV - CP $-\underline{\text { W }}$...... Heat-seeking equipment - (SIC Code - 3829)

- TAM - ME - GZ ...... Airborne remote sensing systems - (SIC Code - $\underline{3829)}$

- $\underline{\text { TAM }}-\underline{\mathrm{SS}}-\underline{\mathrm{FH}}$....... Heat-seeking equipment - (SIC Code $-\underline{3669)}$

- TEL - BR - TEIPS ...... Remote sensing systems - (SIC Code - $\underline{3663)}$ 


\section{Gerardo International, Inc.}

- AKA: MRX Technologies, Inc.

- AKA: MRX Scientific Foundation

- AKA: International Health Trust

\section{Address:}

112 Water Turkey Ct.

Daytona Beach, EL 32119

Tel: (904) 756-1916

Fax: (904) 756-4300

\section{Internet:}

Explore: Resource Pages

HomePage: http://www.mrx.com

e-mail: gerardo@mrx.com

\section{Status:}

Public U.S. company (Ticker symbol: MRX). Formed in 1976.

Government Contractor: Prime

Size:

- Empl-7,800 employees; $99 \%$ employment growth in the year prior to interview.

- Sales - Annual sales $=\$ 672$ million; over $25 \%$ of sales are from international business.

\section{Execs:}

- Neil Gerardo, COB/CEO/President (Chairman, C.E.O.)

- George W. Williams, VP of Marketing (Sales, Marketing)

- Robert Gerardo, Chief Financial Officer (Finance)

- Andrew Cohen, Chief Information Officer (M.I.S.)

- George Bowen, Director of Advanced Research Projects (R\&D)

- Richard T. Lopez, Director of International Sales (Int'l Sales \& Mktg.)

- Ms. Dorothy E. Talley, VP of Public Relations

Primary Industry: Medical (MED)

Primary SIC Code: 8000

Developer of MRX, a virtual technology with transforming applications in medicine, the environment, chemical processing, desalination, on site radioactive remediation, remote sensing and defense. MRX provides the ability to degrade any targeted molecular structure, remotely, by way of an absorption technique. MRX MEDICAL couples and directs magnetic and photonic energy to detect and destroy targeted viruses, bacteria, fungi, cancers and tumors in vivo without side effects. MRX ENVIRONMENTAL couples and directs photonic energies to fully degrade all hazardous organic and inorganic contaminates in water, soil and air. MRX CHEMICAL couples and directs magnetic and photonic energies to catalyze and control the reactions of organic, inorganic, petrochemical and polymer processes. MRX DESALINATION couples and directs magnetic and photonic energies to separate drinking water from sea water in ultra high volumes. Manufacturer of 3-dimensional molecular memory computer chips; remote geological sensors; non-proliferation conventional weapons; remote electronic negation systems; non-proliferation nuclear weapons; and air defense systems. Provider of remote geological sensing, remote nuclear, chemical and biological weapons negation. Provider of anti-personal countermeasures, anti-armor countermeasures, remote communications negation, remote satellite negation, nuclear waste remediation, radioactive waste remediation and pharmaceutical nrocesses. Products and services are sold to the medical. defense. environmental. 
computer, energy, geological, desalination, radioactive waste, pharmaceutical and financial services industries.

\section{Product Codes:}

- $\underline{\mathrm{BIO}}-\underline{\mathrm{IM}}-\underline{\mathrm{B}}$....... Virology systems - (SIC Code - $\underline{2836}$ )

- $\underline{\mathrm{BIO}}$ - IM - $\underline{\mathrm{C}}$...... A Anticancer systems - (SIC Code - 2836)

- $\underline{\mathrm{CHE}}$ - $\underline{\mathrm{SV}}-\underline{\mathrm{R}}$...... Chemical R\&D services - (SIC Code - $\underline{8731}$ )

- $\underline{D E F}-\mathrm{EW}-\mathrm{B}$...... Remote electronic negation systems - (SIC Code - $\underline{3812)}$

- $\underline{D E F}-\underline{E W}-\underline{C L} \ldots . . . \mathrm{MRX}$ remote warhead negation systems - (SIC Code - 3812)

- $\underline{D E F}-\underline{\mathrm{OR}}-\mathrm{YG} \ldots .$. . Air defense systems - (SIC Code - 3812 )

- DEF - $\underline{\mathrm{OR}}-\mathrm{YW}$....... Non-proliferation nuclear weapons - (SIC Code - $\underline{3489}$ )

- $\underline{\mathrm{DEF}}-\underline{\mathrm{OR}}-\underline{\mathrm{YW}} \ldots . .$. Non-proliferation conventional weapons -. (SIC Code - 3489 )

- DEF - ST .......MRX remote electronic negation systems - (SIC Code - $\underline{3812)}$

- ENV - SV - AA ...... Air quality testing services - (SIC Code - 8734)

- ENV - SV - AS ...... Soil analysis services - (SIC Code - 8734)

- ENV - SV - WD ...... Waste disposal services - (SIC Code - $\underline{\text { 4953) }}$

- $\underline{E N V}-\underline{\mathrm{SV}}-\underline{\mathrm{WT}} \ldots . .$. Radioactive waste remediation services - (SIC Code - 4959)

- $\underline{\text { ENV }}$ - $\underline{\text { SV }}$ - $\underline{\text { WT }}$...... Nuclear waste remediation services - (SIC Code - 4959)

- ENV - TR - STD ...... Waste decontamination equipment - (SIC Code - 3589)

- ENV - TR - WTR ....... MRX desalination systems - (SIC Code - 3589)

- $\underline{\text { MED }}$ - $\underline{\text { DG }}-\underline{\text { IL }}$....... MRX medical imaging systems - (SIC Code - $\underline{3845)}$

- $\underline{M E D}$ - $\underline{\mathrm{SU}}-\underline{\mathrm{XLZ}}$.......MRX medical negation systems - (SIC Code - $\underline{3845)}$

- $\underline{\text { MED }}$ - $\underline{\text { SV }}$ - $\underline{\mathrm{R}}$....... Medical R\&D services - (SIC Code - 8731)

- $\underline{\mathrm{SUB}}-\underline{\mathrm{SE}}-\underline{\mathrm{IN}}$..... . 3-dimensional molecular memory computer chips - (SIC Code - $\underline{3674)}$

- TAM - ME - GP ...... Remote geological sensors - (SIC Code - $\underline{3829}$ )

- TEL - BR - TEIPS ....... MRX remote sensing systems - (SIC Code -

- TRN - GS ...... Air surveillance systems - (SIC Code - 3812) 


\section{Inframetrics, Inc.}

\section{Address:}

16 Esquire Rd.

North Billerica, MA 01862

Tel: (508) 670-5555

Fax: (508) 667-2702

Internet:

Explore: Resource Pages

e-mail: info\%infra@mcimail.com

\section{Status:}

Operating unit of a non-U.S. parent. This operating unit formed in 1975. Ultimate parent is Elbit, Ltd. (country Israel).

\section{Government Contractor: Prime}

Size:

- Empl - 170 employees; $11 \%$ employment reduction in the year prior to interview; no employment change projected for the next fiscal year.

- Sales - \$10m - \$25m annual sales; includes sales from international business.

Execs:

ว J. Teich, President (C.E.O.. R\&D)

$\checkmark$ Andrew Teich. VP. of Sales (Sales)

$\therefore$ Azrie! Biberstain. VP of Finance (Financt. M.I.S.)

$=$ Frank Perry. VP of Business Development (Marketing)

Primary Industry: Telecomununications (TEL)

Primary SIC Code: 3663

Manufacturer and designer of infrared imaging and temperature measurement systems. Products include fadiometers and night vision equipment. Products are sold to multiple industries.

\section{Product Codes:}

- $\underline{\mathrm{PHO}}-\underline{\mathrm{OP}}-\underline{\mathrm{IR}}$...... Radiometers - (SIC Code - $\underline{3826}$ )

- IAM - ME - MT ...... Temperature measuring equipment - (SIC Code - 3822)

- IEL - BR - IEII ...... Night vision equipment - (SIC.Code - 3663) 
Sets Technology, Inc.

\section{Address:}

300 Kahelu Ave., Suite 10

Mililani. HI 96789

Tel: (808) 625-5262

Fax: (808) 625-2474

\section{Internet:}

Explore: Resource Pages

e-mail: sets@sets.hawaii.com

Status:

Independent private U.S. company. Formed in 1976.

Government Contractor: Prime

Size:

- Empl- 40 employees; $33 \%$ employment growth in the year prior to interview; $50 \%$ employment growth projected for the next fiscal year.

- Sales - $\$ 2.5 \mathrm{~m}$ - $\$ 5 \mathrm{~m}$ annual sales; $10 \%$ to $25 \%$ of sales are from international business.

\section{Execs:}

- Nick Susner, President/CEO (C.E.O., Sales, R\&D)

- Courtney Theupa, Controller (Finance, Purchasing)

- Greg Mooradian, Executive Vice President

Primary Industry: Telecommunications (TEL)

Primary SIC Code: 3663

Research and development organization specializing in remote sensing and image processing. Research is performed primarily for the government, but also for some commercial markets.

\section{Product Codes:}

- $\underline{\text { TEL }}$ - BR - TEIPD ...... Image processing research and development - (SIC Code - 3663 )

- TEL - BR - TEIPS ...... Remote sensing research and development - (SIC Code - 3663)

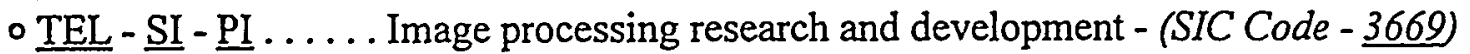

- TEL - SV - - ...... Image processing research and development services - (SIC Code - 8731)

- $\underline{\mathrm{TEL}}-\underline{\mathrm{SV}}-\underline{\mathrm{R}}$...... Remote sensing research and development services - (SIC Code $-\underline{8731}$ ) 


\section{Sp̈ectron Engineering, Inc.}

\section{Address:}

255 Yuma Ct.

Denver, $\mathrm{CO} 80223$

Tel: (303) 733-1060

Fax: (303) 733-2432

\section{Internet:}

Explore: Resource Pages

Status:

Independent private U.S. company. Formed in 1981.

Government Contractor: Sub-contract

Size:

- Empl - 10 employees; $42 \%$ employment growth in the year prior to interview; no employment change projected for the next fiscal year.

- Sales - Estimated annual sales $=\$ 1.4$ million; $2.5 \%$ to $10 \%$ of sales are from international business .

\section{Execs:}

- Norman Herz, President (C.E.O., Finance)

- Frosty Claypool, Production Manager (R\&D, Engineering, Manufacturing)

- Roger Sweeney, Office Manager (Administration)

\section{Primary Industry: Factory Automation (AUT)}

Primary SIC Code: 3569

Manufacturer of spectroradiometers, microdensitometers, inspection equipment and CRT analysis equipment. Provider of custom machine vision systems design services. The spectroradiometers and microdensitometers'are used to measure colors in the entire spectrum and are used in remote sensing applications. The company also manufactures IR cameras which are electro-optic test equipment used in measuring light. Inspection equipment is used for parts gauging and measurement for size and shapes of small objects. CRT analysis equipment is used to - measure and analyze CRT display projections. Products and services are sold to the aerospace and environmental industries. This company was capitalized by private investment.

\section{- Product Codes:}

- $\underline{\mathrm{AUT}}-\underline{\mathrm{AT}}-\underline{\mathrm{O}} \ldots . . . \mathrm{CRT}$ analysis equipment - (SIC Code - $\underline{\text { 3829)}}$

- $\underline{\text { AUT }}-\underline{\mathrm{MV}}-\underline{\mathrm{C}}$....... Custom machine vision systems - (SIC Code $-\underline{3569}$ )

- AUT - MV -1 I..... Parts gauging inspection systems - (SIC Code $-\underline{3569)}$

- $\underline{\mathrm{AUT}}-\underline{\mathrm{SV}}-\underline{\mathrm{M}}$....... Custom machine vision system design services - (SIC Code $-\underline{8711)}$

- $\underline{\mathrm{PHO}}-\underline{\mathrm{CA}}-\underline{\mathrm{M}}$....... IR cameras - (SIC Code $-\underline{3861}$ )

- $\underline{\mathrm{PHO}}-\underline{\mathrm{OP}}-\underline{\mathrm{IR}}$...... Spectroradiometers - (SIC Code - $\underline{3826})$

- $\underline{\text { TAM }}$ - $\underline{\mathrm{AN}}-\underline{\mathrm{E}}$...... . Spectral analyzers - (SIC Code - $\underline{3825}$ )

- TAM - ME - $\underline{\mathrm{P}}$...... . Photo-optical measuring equipment - (SIC Code - $\underline{3829}$ )

- TEL - BR - TEIPS ...... Microdensitometers - (SIC Code - 3663) 


\section{Diode Lasers}

Open Path and Insitu 


\section{Bov̀ar, Inc.}

\section{Country: Canada}

\section{Internet:}

Explore: $\underline{\text { Resource Pages }}$

\section{Status:}

Non-U.S. parent company.

Primary Industry: Environmental (ENV)

Parent/holding company with a high-tech operating unit in the test and measurement industry.

\section{Product Codes:}

$\circ \underline{\mathrm{ZZZ}}-\underline{\mathrm{HC}} \ldots . .$. Parent/Holding company - (SIC Code $-\underline{6719})$

\section{Units:}

The following single operating unit involved in high-tech is listed below, and is profiled in the CorpTech Directory of Technoiogy Companies.

- Spectrum Diagnostix. Inc., MA 


\section{Spectrum Diagnostix, Inc.}

- FKA: PSI Environmental Instruments Corp.

\section{Address:}

20 New England Business Ctr.

Andover, MA 01810

Tel: (508) 686-8801

Fax: (508) 686-8809

\section{Internet:}

Explore: $\underline{\text { Resource Pages }}$

\section{Status:}

Operating unit of a non-U.S. parent. This operating unit formed in 1992. Ultimate parent is Bovar, Inc. (country Canada).

Government: Subcontractor

Size:

- Sales - $\$ 2.5 \mathrm{~m}$ - $\$ 5 \mathrm{~m}$ annual sales; $10 \%$ to $25 \%$ of sales are from international business.

- Empl - 18 employees; no employment change in the year prior to interview.

\section{Execs:}

- Robert Weiss, Chairman of the Board (Chairman)

- Patrick McDonnell, President/CEO (C.E.O.)

- Roy Langlois, VP of Manufacturing and Operations (Manufacturing)

- Michael B. Frish, VP of Product Development (R\&D)

- James E. Staudt, VP of Business Development (Corporate Dev., Marketing)

- Stephen Genestreti, Chief Financial Officer (Finance)

Primary Industry: Environmental (ENV)

Primary SIC Code: 3829

Manufacturer of continuous, on-line emissions monitors for control of various fugitive gas engines. Products are sold to the utility, chemical, petrochemical and metal processing industries. This company was capitalized by private investment and venture capital.

\section{Product Codes:}

- $\underline{E N V}-\underline{A N}-\underline{G} \ldots .$. . Continuous emissions monitors - SpectraScan ${ }^{\mathrm{TM}}$ Perimeter - (SIC Code $\left.-\underline{3823}\right)$

- $\underline{E N V}-\underline{A N}-\underline{G} . . .$. . Continuous emissions monitors - SpectraScan ${ }^{\mathrm{TM}}$ Ammonia - (SIC Code - $\underline{3823}$ )

- $\underline{E N V}$ - $\underline{\mathrm{AN}}-\underline{\mathrm{G}} . . .$. . Continuous emissions monitors - SpectraScan ${ }^{\mathrm{TM}}$ Multi Pollutant - (SIC Code $-\underline{3823}$ )

$\circ \underline{E N V}-\underline{\text { CP }}-\underline{A} \ldots . .$. Continuous emissions monitors - GasTemp ${ }^{\mathrm{TM}}-($ SIC Code $-\underline{3564)}$

- TAM - PV - E ...... Continuous emissions monitors - GasTemp ${ }^{\mathrm{TM}}-($ SIC Code $-\underline{3823})$ 


\section{Southwest Sciences, Inc.}

\section{Address:}

1570 Pacheco St., Suite E-11

Santa Fe, NM 87505

Tel: (505) 984-1322

Fax: (505) 988-9230

\section{Internet:}

Explore: Resource Pages

HomePage: http://www.iac.net

e-mail: sws@rt66.com

\section{Status:}

Independent private U.S. company. Formed in 1985.

Government: Prime Contractor

Size:

- Sales - Estimated annual sales $=\$ 2.3$ million; no international sales.

- Empl- 9 employees; $12 \%$ employment growth in the year prior to interview; no employment change projected for the next fiscal year.

\section{Execs:}

- Alan Stanton, President (C.E.O., Sales, M.I.S.)

- Joel Silver, Vice President (R\&D, Finance)

Primary Industry: Chemicals (CHE)

Primary SIC Code: 8700

Provider of research and development services in physical chemistry, optics and laser applications, for such purposes as improvements in gas detection, or diagnosis of combustion and chemical processes. This company was capitalized by private investment.

\section{Product Codes:}

- CHE - $\underline{\text { SV }}-\underline{\mathrm{R}}$...... Physical chemistry R\&D services - (SIC Code - 8731)

- $\underline{\mathrm{PHO}}-\underline{\mathrm{SV}}-\underline{\mathrm{R}}$..... . Optics and laser R\&D services - (SIC Code - 8731)

- $\underline{\text { TAM }}$ - $\underline{\text { SV }}$...... Gas detection R\&D services - (SIC Code - 1734 ) 


\section{for September 1997}

Earth Vision is a virtual environmental community (uww.earthvision.net) providing news, information, discussion forums and more across the areas of: business, technology, policy. education, recreation, and sustainability. Below are summary statistics on Earth Vision's audience profile, activity level, and growth trends. Statistics were produced by interse' Market Focus ${ }^{\mathrm{TM}}$ Web Analysis software. Questions regarding this report should be directed to Stuart Claggett, Vice President of Environmental Programs for Global Enviror:ment \& Technology Foundation at (703) 750-6401.

\section{AU⿴囗IENCE PROFILE}

User Groups by Geographic Location

United States

International

$79 \%$

$21 \%$

User Groups by Organization (U.S. Only)

Small Industry

Medium-Large Industry

Non-Profit

Academic

Govemment

Military

$33 \%$
$49 \%$
$2 \%$
$9 \%$
$6 \%$
$1 \%$

Top 10 Countries
1. United States
6. Israel
2. Canada
3. Australia
4. United Kingdom
5. Japan
8. South Korea
9. New Zealand
10. Thailand
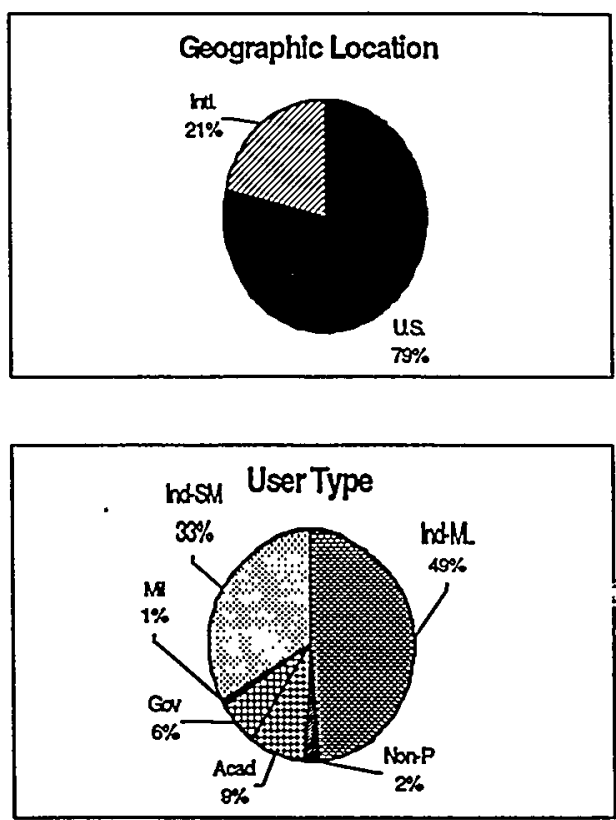

\section{ACTIVITY LEVEL}

Number of Hits Recorded

Number of Kilobytes Transferred Daily
440,013

$1,650,000$

\section{GROWTH TRENDS}

Increase in Hits from Previous Month

increase in Registered Users from Previous Month

Increase in Daily Visitors from Previous Month .

Increase in Kilobytes Transferred from Previous Month
$-15 \%$

$17 \%$

$3 \%$

$-7 \%$

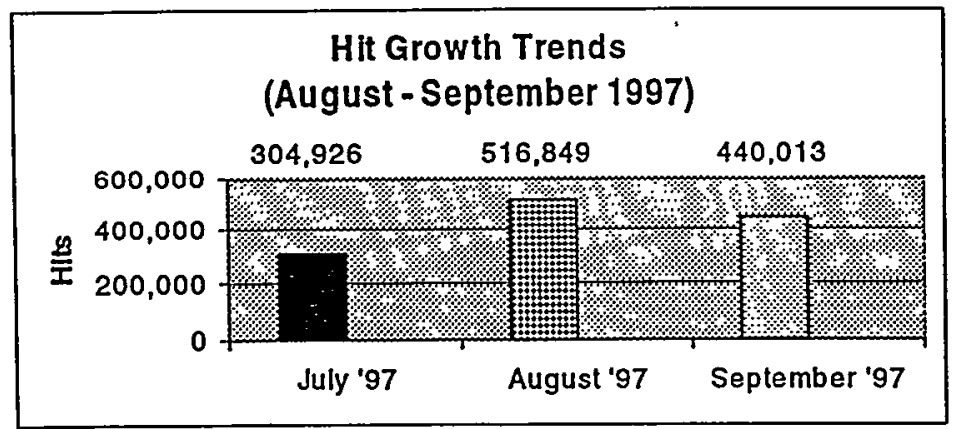




$\begin{array}{ll}\text { From: } & \text { Michelle Batten } \\ \text { Sent: } & \text { Friday, September 05, 1997 1:55 PM } \\ \text { To: } & \text { 'techknow-l@gxinet.com' } \\ \text { Subject: } & \text { GNET Weekly Highlights }\end{array}$

GNET Weekly Highlights - Friday, September 5, 1997 Edition

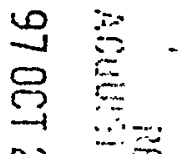

Welcome TechKnow users to the improved e-mail bulletin, now called "GNET Weekly Highlights." The new upon us bringing fresh opportunities and challenges. Be sure to share your experiences with us so others can learin!more. Techknow is an excellent place to post case studies of how your innovative technology is providing clean sojutions -Visit TechKnow today at http://www.gnet.org/forsite/?SubSystemID=1\&ComponentID=4936 and add your latest tupdates?

SITE ROUNDUP



Catch the Front Page News at hitp://www.gnet.org !

- DOE Awards $\$ 46$ Million For Grants to Speed Environmental Cleanup

- Brookhaven Lab's New Degreasing Facility Prevents Pollution

- ERHC And Air Group To Build First Titan Oil \& Tire Recycling Plant In Louisiana

- Biodegradation of TCE through Toluene Injection

Stay informed with the GNET News Center at http://www.gnet.org/forsite/?SubSystemID=1\&ComponentID=3406 - Enviro-Innovations, August 1997 in the "Journals" section is a compilation of selected news items providing recent information on the Canadian environmental industry

Get a jump on business with the GNET Market Center at http://www.gnet.org/forsite/?SubSystemID=1\&ComponentID= 2483

- The National Center for Environmental Research and Quality Assurance (NCERQA) will be announcing its remaining Fiscal 1998 Science to Achieve Results (STAR) Program Grants!

- New Section: Doing Business With.... Information for businesses wishing to work cooperatively with federal or state sources for licensing opportunities, technology solutions and technology transfer.

- New! Characterization Technology for Radioactively Contaminated (TRU) Surfaces Needed. Check out this week's Contracting Opportunities!

Learn about new.developments in the GNET Technology Center at http://www.gnet.org/forsite/?SubSystemID=1

\&ComponentID $=4523$

- Plume Dating Combines Science with Good Detective Work

- TechKnow now contains over 1100 technology profiles!

Check out federal, state, and local regulatory progress with the GNET Government Center at http://www.gnet.org/forsite/? SubSystemID $=1 \&$ ComponentID $=1710$

- Case Studies of Selected States ${ }^{2}$ Voluntary Cleanup/Brownfields Programs available at the ITRC Home Page under

"reports." Go to the "Interstate Cooperation Initiatives" section to view and send your comments to fmurray@getf.org.

Get Focused with the DOE/GETE Center at http://www. gnet.org/forsite/?SubSystemID=1\&ComponentID=4331 - Featured: In Situ Oxidation Destruction of DNAPL

- Tanks Focus Area Page: Do you know how much time is left to remove or upgrade your underground tanks?

EV BUSINESS \& TECHNOLOGY BULLETIN....

Don't forget to stop in and visit this Earth Vision neighborhood at http://www.earthvision.net! This week:

- Special Section on Tanks: Was your underground storage tank (UST) installed before December 22, 1988?

- Environmental Business Council Resources Report (Program and Contact info included for 28 organizations)

- EV Partner "TSD Central" offers listing of business opportunities active in the environmental management industry or list a business opportunity of your own.

TECHKNOW SPOTLIGHT.....

$A C T^{\star} D E^{\star} C O N$ is a dilute, aqueous based, environmentally non-hazardous technology to selectively dissolve radioactive contaminants and certain heavy metals from soil or other materials. The process can be applied either in-situ or ex-situ. Once dissolved, the contaminates are recovered by either ion exchange, evaporation, or the MAG* SEP process (TechKnow profiled technology), leaving clean soil or other treated materials for free release. The primary benefit of this technology is that by selectively dissolving the contaminant materials, large volumes of soil can be released, while the waste volume will contain only the contaminants 
removed and the dissolution salts. For more information, go to TechKnow at http://www.gnet.org/forsite/?SubSystemiD=1 \&.ComponentlD $=4936$

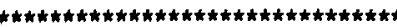 \\ CONFERENCE HOT LIST.....}

- Financing Technology: The "Bottom Line" on Manufacturing Efficiency Monday, September 22, 1997, Chicago, IL one-day workshop showcases financing of energy-efficient manufacturing technologies

- For The CEO of the 1990's: Risk-Based Decision-Making Influencing Financial Transactions and Brownfield Redevelopments

September 30 and October 1, 1997, Chicago, IL

The purpose of the conference is two-fold: One to lay out a framework for building a streamlined and technically

defensible "risk-based corrective action" program for mandatory and voluntary site cleanups and two, to apply a risk-based cleanup program in financial transactions and brownfield redevelopments.

For details and contact information go to GNET Home Page and News Center.

SPEAK OUT!......

One of our users recently asked this question about treating hazardous waste, "Company I work for has found a method to chemically treat mop water. We are a large quantity generator of hazardous waste. Can we treat the mop water without being a treatment facility? Do we need to file a permit for this first?"

Any suggestions? Post your suggestions in the GNET message board via Earth Vision!

This is a moderated listserv message. Replies should be e-mailed to gnet@getf.org.

Produced by the Global Environment \& Technology Foundation for the Global Network of Environment \& Technology (GNET)

Visit GNET at http://www.gnet.org 


\section{Byrne, Tina}

\section{From:}

Sent:

To:

Subject:
Michelle Batten

Monday, September 15, 1997 10:08 AM

'techknow-l@gxinet.com'

GNET Weekly Highlights - September 15, 1997

\section{GNET Weekly Highlights - Monday, September 15, 1997 Edition}

GNET is exploding with information! Newly acquired links and reports from international sources are available throughout the site - let us know what you want to see! E-mail us at gnet@getf.org.

SITE ROUNDUP

Catch the Front Page News at http://www. gnet.org!

- Argonne, Australian Group To Work On Radwaste Compression Technology

- North American Environmental Commission Releases Air Pollution Report

- New Membranes Could Expand Gas Separation Applications

- Korea Dioxin Control Technology Fair

Stay informed with the GNET News Center at http://www.gnet.org/forsite/?SubSystemID =1\&ComponentlD =3406 - August 1997 issue of "GroundWater Currents" which covers developments in innovative groundwater treatment is now available!

- Hot Press Release! Westinghouse Wins $\$ 35$ Million Spent Nuclear Fuel System Contract

- Get a jump on business with the GNET Market Center at http://www,gnet.org/forsite/?SubSystemID=1\&ComponentID= 2483

- Superfund Insurance Problems Dog Business

- Links to International and U.S. Business Incubators Now Updated!

Learn about new developments in the GNET Technology Center at http://www.gnet.org/forsite/?SubSystemID=1

\&.Component $\mid \mathrm{D}=4523$

- Featured: Technical Overview on In Situ Flushing

- 200 new technology profiles added to TechKnow!

Check out federal, state, and local regulatory progress with the GNET Government Center at http://www.gnet.org/forsite/? SubSystemiD=1\&ComponentiD $=1710$

- Technology Partnering - Can you Count on Government?

- In the "State Government" section, "On Common Ground: Sustainable Community Activities and Pollution Prevention"

Get Focused with the DOE/GETE Center at http://www.gnet.org/forsite/?SubSystemID=1\&ComponentID=4331

- Featured: A Promising New Technology for Treating DOE's Spent Nuclear Fuels

- - SubCon Focus Area Page: Measuring Fuel Contamination Using High Speed GC and Cone Penetration Techniques

\section{EV BUSINESS \& TECHNOLOGY BULLETIN....}

Don't forget to stop in and visit this Earth Vision neighborhood at http:/lwww.earthvision,net! This week:

- Learn about the fastest growing companies in the environmental industry!

- EV Partner "Greenwave Radio" interviews the Leesburg, VA Superintendent of Water Pollution Control about their innovative treatment initiatives.

TECHKNOW SPOTLIGHT.....

$A C T^{\star} D E^{\star} C O N$ is currently featured on TechKnow! Your technology could be next! Submit your technology nominations to gnet@gelf.org to have your technology spotlighted on our high traffic database. 
This course stresses the underlying concepts and field implementation procedures for evaluating and applying remediation by natural attenuation.

- Southern States Annual Environmental Conference

September $23-35,1997$, Biloxi, MS

Topics of interest ranging from pollution prevention in air, water, land, new environmental regulations, current issues of interest in waste management or waste minimization.

- Breaking Into Overseas Markets

September 29, 1997, Williamsburg, VA

Virginia's Center for Innovative Technology and the Virginia Economic Development Partnership are sponsoring a workshop designed to position your company for entry into the global market for environmental services and products.

For details and contact information go to GNET News Center and click on the "Calendar of Events" for listings.

SPEAK OUT!......

This past week GNET featured remarks from Jack Gibbons, the Assistant to the President on Science \& Technology, regarding the success of technolocy partnering between industry and government. Do you agree that these partnerships work? What steps would you recommend to others in order to develop technology partnerships? E-mail your comments and share your experiences with us at gnet@getf.org!

This is a moderated listserv message. Replies should be e-mailed to gnet@getf.org.

Produced by the Global Environment \& Technology Foundation for the Global Network of Environment \& Technology (GNET).

Visit GNET at http://www. gnet.orc 
From:

Michelle Batten [michelle.batten@getf.org]

Sent:

Monday, September 22, 1997 11:22 AM

To:

Subject:

'techknow-l@gxinet.com'

GNET Weekly Highlights

GNET Weekly Highlights - Monday, September 22, 1997 Edition

Want to work cooperatively with federal or state sources for licensing opportunities, technology

solutions and technology transfer? Check out our "Doing Business

With.." section at

http://www.gnet.org/forsite/?SubSystemID=1\&ComponentID=17021, located in

the GNET Market Center. You'll find a wealth of links to DOE, DOC, the

Army, and more who are looking to partner with the private sector on a variety of technology initiatives.

SITE ROUNDUP.

Catch the Front Page News at http://www.gnet.org !

- Science Summit on Climate Change

- Incentive Payments Paid For Generating Clean Energy

- Pollution Prevention 1997: A National Progress Report

Stay informed with the GNET News Center at

http://www.gnet.org/forsite/?SubSystemID=1\&ComponentID=3406

- Studies Focus on Reducing Lead Bioavailability at Superfund Site -

Check out the July issue of "Tech Trends" in the Journals section.

- Hot Press Release! Peña Announces New Effort To Develop Advanced

Methods To Sequester Or Recycle Greenhouse Gases

Get a jump on business with the GNET Market Center at

http://www.gnet.org/forsite/?SubSystemID=1\&ComponentID=2483.

- The United States Experience With Economic Incentives In

Environmental Pollution Control Policy

- Partnering with Allied Signal Aerospace's Kansas City Plant (KCP) in

"Doing Business With..."

Learn about new developments in the GNET Technology Center at

http://www.gnet.org/forsite/?SubSystemID $=1$ \&ComponentlD $=4523$

- Featured: Heavy Metal Meets Its Match -Two new materials strip pollutants from toxic wastes

making hazardous wastewater "clean enough to drink."

- Check out the INEEL Priorities List for technology development in

"Tech Needs"

Check out federal, state, and local regulatory progress with the GNET

Government Center at

http://www.gnet.org/forsite/?SubSystemID=1\&ComponentID=1710

- Clean Sites Innovative Technology Program

- Links to international law resources available in the "Law Library."

Get Focused with the DOE/GETE Center at

http://www.gnet.org/forsite/?SubSystemlD $=1$ \&ComponentlD $=4331$

- Featured: A Promising New Technology for Treating DOE's Spent Nuclear

Fuels

- Mixed Waste Focus Area Page: Check out the Microwave Melter.....

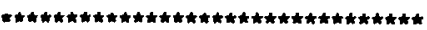

EV BUSINESS \& TECHNOLOGY BULLETIN....

Don't forget to stop in and visit this Earth Vision neighborhood at

http://www.earthvision.net! This week:

- ABC's of Pollution Prevention for Small Businesses 
- Add your company to our free Business Directory!

\section{TECHKNOW SPOTLIGHT....}

Several of you have asked us what information is required in order to have technology profiles approved for public viewing on TechKnow. Below are a set of guidelines for your reference that will also be posted within TechKnow. As always, please forward any questions or comments to gnet@getf.org.

The following information fields must be completed:

- Technology Name (must be full technology name)

- Keywords (choose at least 3)

- Media (choose at least 1)

- Contaminants (choose at least 1)

- Summary (3-5 sentence description of technology)

- Long Description (1-2 paragraphs on technology and what it can do)

- Status (choose one from the pull down menu)

- Cost (at least provide price range)

- Limitations (3-5 sentences)

- Tech Need (3 -5 sentences on the need for the technology)

- Contacts (must supply at least one point of contact with company name, phone, fax. Supply e-mail address and Web site if available.)

**Before sending your profile to the moderator for approval, be sure to check for spelling errors. Text should follow basis rules of grammar.

- Science Summit on Climate Change September 30, 1997, Washington, DC

With the crucial Kyoto negotiations just a few months away, the event offers a unique opportunity for scientists and other citizens to learn about and discuss the current state of scientific knowledge on climate change.

\section{Call for Energy and Environmental Technologies}

10/1/1997, New York, NY

- The Environmental Business Association of NYS and the New York State

- Energy Research and Development Authority (NYSERDA) invite technology developers to submit abstracts describing emerging technologies that will improve environmental or energy system quality, efficiency, or conservation.

- Fundamentals Of The Contaminant Hydrogeolgy Of Fractured RocksImplications For Remediation

$09 / 30 / 1997-10 / 02 / 1997$

This intense 20 hour course is aimed at practicing hydrogeologists, engineers, environmental scientists and site managers who are faced with the difficulties of investigating and remediating groundwater contamination in fractured rock.

For details and contact information go to GNET News Center and click on the "Calendar of Events" for listings.

SPEAK OUT!......

Mike Barnett is trying to develop a position at my Power Plant that will allow a Water Treatment Plant Operator perform sampling and analysis that will be considered acceptable (certified) by the EPA or related organization (POTW,etc.) In order to get a sufficient pay scale, he needs to prove that a degree or equivalent is needed. Does anyone know of an authoritative source (document nublication rearulation 
From:

Batten, Michelle [michelle.batten@getf.org]

Sent:

Monday, September 29, 1997 2:08 PM

To:

'techknow-l@gxinet.com'

Subject:

GNET Weekly Highlights

GNET Weekly Highlights - Monday, September 29, 1997 Edition

GNET is exploding with information! Newly acquired links and reports

from international sources are available throughout the site - let us know what you want to see! E-mail us at gnet@getf.org.

SITE ROUNDUP

Catch the Front Page News at http://www.gnet.org !

- NREL Researchers Test Solar Thermal Technology

- Clean Technology Can Achieve Significant Greenhouse Gas Reductions

Stay informed with the GNET News Center at

http://www.gnet.org/forsite/?SubSystemID=1\&ComponentID=3406

- Check out the Daily Regulatory Reporter: Includes a daily summary of

EPA, OSHA and DOT (Environmental related) regulations as published the

day before in the Federal Register.

- In the Press Box: Hammer Facility Adds New Dimension To Safety

Training At Hanford

Get a jump on business with the GNET Market Center at

http://www.gnet.org/forsite/?SubSystemlD=1\&ComponentID=2483

- Top Targets for Trade Promotion

- Contracting Opportunities: Secondary Containment For Oil Storage DUE

092997 and more!

Learn about new developments in the GNET Technology Center at

http://www.gnet.org/forsite/?SubSystemID =1\&ComponentID=4523

- Featured: EPA Environmental Research Brief on Anaerobic

Biodegradation of BTEX in Aquifer Material

Check out federal, state, and local regulatory progress with the GNET

Government Center at

http://www.gnet.org/forsite/?SubSystemID=1\&ComponentID=1710

- National Brownfields Assessment Demonstration Pilots

- In the "Law Library"... The British Columbia Environmental Appeal

Board

Get Focused with the DOE/GETE Center at

http://www.gnet.org/forsite/?SubSystemID $=1$ \&ComponentID $=4331$

- Featured: A Promising New Technology for Treating DOE's Spent Nuclear

Fuels

- CMST Focus Area Page: Process Monitoring and Characterization by

Vibrational Spectroscopies

EV BUSINESS \& TECHNOLOGY BULLETIN....

Don't forget to stop in and visit this Earth Vision neighborhood at

http://www.earthvision.net! This week:

- Integrated Remediation And Financing Program

- Pollution Costing China \$54 Billion Annual Economic Loses

TECHKNOW SPOTLIGHT.....

Techknow is offline due to a maintenance upgrade. The database will be 


$$
\because \text {, }
$$

available after Tuesday, September 30,1997. We appreciate your patience and apologize for any inconvenience.

\section{CONFERENCE HOT LIST.....}

- 11th Annual Conference, Academy of Certified Hazardous Materials Managers

10/05/1997 - 10/08/1997, Atlantic City, NJ

- American Society of Civil Engineers In Situ Remediation of the Geoenvironment (In Situ '97)

10/05/1997 - 10/09/1997, Minneapolis, MN

The conference will focus on the analysis, design, modeling, construction and performance of in situ systems for the remediation of the subsurface environment.

- The Sixth International Conference on Radioactive Waste Management and Environmental Restoration.

10/12/1997 - 10/16/1997, Beijing, China

This conference promotes broad international exchange of information on technologies, operations, management approaches, economics, and public policies in the critical areas or radioactive waste management and environmental remediation.

For details and contact information go to GNET News Center and click on the "Calendar of Events" for listings.

\section{SPEAK OUT!......}

Daniel Berler, an Earth Vision member from Rutgers University asks, "Does anyone know of any good references concerning the failure of pump and treat and/or vacuum extraction remediation?" Post your responses to the Earth Vision Message Board or e-mail him direct at berler@rci.rutgers.edu.

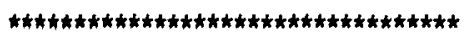

This is a moderated listserv message. Replies should be e-mailed to gnet@getf.org.

Produced by the Global Environment \& Technology Foundation for the Global Network of Environment \& Technology (GNET).

Visit GNET at http://www.gnet.org 